\title{
Fair valuation of insurance liability cash-flow streams in continuous time: Applications
}

\author{
Łukasz Delong ${ }^{1}$, Jan Dhaene ${ }^{2 *}$, Karim Barigou ${ }^{2 *}$ \\ ${ }^{1}$ Warsaw School of Economics \\ Institute of Econometrics, Department of Probabilistic Methods, \\ Niepodległości 162, Warsaw 02-554, Poland, \\ lukasz.delong@sgh.waw.pl \\ ${ }^{2} \mathrm{KU}$ Leuven \\ Actuarial Research Group, AFI, Faculty of Business and Economics, \\ Naamsestraat 69, 3000 Leuven, Belgium, \\ jan.dhaene@kuleuven.be \\ karim.barigou@kuleuven.be
}

*Jan Dhaene and Karim Barigou acknowledge the financial support of the Onderzoeksfonds KU Leuven (GOA/13/002). Karim Barigou is a PhD fellow of the Research Foundation - Flanders (FWO) [grant number $1146118 \mathrm{~N}]$. 


\begin{abstract}
Delong et al. (2018) presented a theory of fair (market-consistent and actuarial) valuation of insurance liability cash-flow streams in continuous time. In this paper, we investigate in detail two practical applications of our theory of fair valuation. In the first example, we consider the fair valuation of a terminal benefit which is contingent on correlated tradeable and non-tradeable financial risks. In the second example, we consider a portfolio of unit-linked contracts contingent on a non-tradeable insurance and a tradeable financial risk. We derive partial differential equations (PDEs) which characterize the continuous-time fair valuation operators in these two examples and we find explicit solutions to these PDEs. The fair values of the liabilities are decomposed into the best estimate of the liability and a risk margin. The arbitrage-free representations of the fair values of the liabilities are derived and the dynamic hedging strategies associated with the continuous-time fair valuation operators are also established. Detailed interpretations of the results, which should be useful both for researchers and practitioners, are provided.
\end{abstract}

Keywords: Optimal quadratic hedging, actuarial valuation, market-consistent valuation, partial differential equation, best estimate, risk margin, net asset value. 


\section{Introduction}

In Delong et al. (2018) we presented a theory of fair (market-consistent and actuarial) valuation of insurance liability cash-flow streams in continuous time. We first considered oneperiod hedge-based valuations, where in the first step, an optimal dynamic hedge for the liability is set up, based on the assets traded in the market and a quadratic hedging objective, while in the second step, the remaining part of the claim is valuated via an actuarial valuation. Then, we extended this approach to a multi-period setting by backward iterations for a given discrete-time step $h$, and considered the continuous-time limit for $h \rightarrow 0$. We formally derived a partial differential equation for the continuous-time valuation operator and proved that this valuation operator is actuarial and market-consistent. We showed that our continuous-time fair valuation operator has a natural decomposition into a best estimate of liability and a risk margin.

In this paper, the main results of our theory of continuous-time fair valuation are illustrated with two detailed examples. In the first example, we consider the fair valuation of a terminal benefit which is contingent on correlated tradeable and non-tradeable financial risks. In the second example, we consider a portfolio of unit-linked contracts contingent on a non-tradeable insurance and a tradeable financial risk. We derive partial differential equations (PDEs) which characterize the continuous-time fair valuation operators in these two examples and we find explicit solutions to these PDEs. The fair values of the liabilities are decomposed into the best estimate of the liability and a risk margin. The arbitrage-free representations of the fair values of the liabilities are also derived. The dynamic hedging strategies associated with the continuous-time fair valuation operators are established. Detailed interpretations of the results are provided. We believe that this paper should be of interest both for researchers and practitioners in actuarial science.

In insurance practice we use at least two valuation standards: Solvency II and IFRS 17. Both valuation standards use the terms called best estimate and risk margin (risk adjustment). These terms are well-understood in practice. However, they have not been formalized from mathematical point of view and the research how to define a valuation rule that leads to a best estimate and a risk margin is still going on. The best estimate corresponds to the expected value (unbiased probability-weighted estimate) of the future cash-flows discounted with an interest rate to reflect the time value of money. The risk margin (risk adjustment) is added for non-hedgeable risks in order to protect the insurer from adverse deviation in the non-hedgeable risks or to compensate the insurer for taking the non-hedgeable risks. In Solvency II the risk margin is derived by calculating the $99.5 \%$-Value-at-Risk of the change in the net asset value in one year time horizon, projecting the Value-at-Risk measures for the next years till maturity of the portfolio, discounting the one-year Value-at-Risk mea-

sures and applying the cost of capital rate. Our valuation model works in the same way but, 
since we consider a continuous-time model, we introduce a continuous-time (instantaneous) solvency capital requirement which is continuously integrated in order to derive the total risk margin for the liability. Our approach can be useful for understanding the valuation concept in Solvency II. Moreover, our approach can be used for valuation of insurance contracts under IFRS 17 where the risk adjustment is a company-specific perception on the non-hedgeable risks and can be freely defined by the insurance company. Since in IFRS 17 the risk adjustment results from indifference pricing of the variable liability cash-flows, the risk adjustment can be related to continuous-time solvency capital requirements if the shareholders decide to provide the capital on a continuous basis to cover the non-hedgeable losses and ask for a continuous-time compensation for bearing the non-hedgeable risks. We believe that continuous measuring of hedgeable and non-hedgeable risks and updating the capital requirement for the non-hedgeable risks on higher frequency than annual is a good direction in valuation and solvency requirements.

The remainder of this paper is structured as follows. In Section 2 we describe the financial and insurance model and we introduce the two examples. Section 3 illustrates the one-period valuation operator and the optimal hedging strategy for the liability by quadratic hedging. Section 4 focuses on the continuous-time fair valuation operator. Finally, Section 5 provides some numerical illustrations of our valuation framework.

\section{$2 \quad$ Financial and Insurance Model}

\subsection{The financial market}

We consider a Black-Scholes financial model. The financial market consists of a risk-free asset $R=(R(t), 0 \leq t \leq T)$ and two risky assets: $Y=(Y(t), 0 \leq t \leq T)$ and $F=(F(t), 0 \leq$ $t \leq T)$. The price of the risk-free asset grows exponentially:

$$
\frac{d R(t)}{R(t)}=r d t, \quad 0 \leq t \leq T, \quad R(0)=1
$$

We assume that the prices of the risky assets $Y$ and $F$ are modelled with correlated geometric Brownian motions and follow the dynamics

$$
\begin{aligned}
& \frac{d Y(t)}{Y(t)}=\mu_{Y} d t+\sigma_{Y} d W_{Y}(t), \quad 0 \leq t \leq T, \quad Y(0)=y_{0}, \\
& \frac{d F(t)}{F(t)}=\mu_{F} d t+\sigma_{F} d W_{F}(t), \quad 0 \leq t \leq T, \quad F(0)=f_{0},
\end{aligned}
$$

where $\mu_{Y}, \mu_{F}, \sigma_{Y}, \sigma_{F}$ denote the drifts and volatilities of the risky assets and $W_{Y}, W_{F}$ denote two correlated Brownian motions with correlation coefficient $\rho$. We define

$$
W_{Y}(t)=W_{1}(t), \quad W_{F}(t)=\rho W_{1}(t)+\sqrt{1-\rho^{2}} W_{2}(t), \quad 0 \leq t \leq T,
$$


where $W_{1}, W_{2}$ are two independent Brownian motions.

The insurance company can invest in the risk-free asset $R$ and in the risky asset $Y$. The risky asset $F$ is not available for trading and represents the underlying investment fund for the contracts sold by the insurance company. We can note that the risky asset $F$ has two components: a tradeable component correlated with $Y$ and a non-tradeable component independent of $Y$. The tradeable component of $F$ is modelled with $W_{1}$, and the non-tradeable component of $F$ is modelled with $W_{2}$.

\subsection{The insurance portfolio}

The insurance company holds a homogeneous portfolio which consists of $n$ identical insurance policies issued at the same date. All policyholders have the same age (or are classified to the same age group) at the inception of the policies. Each policyholder is entitled to three types of benefits: a continuous annuity benefit $A$ paid as long as the policyholder is alive, a death benefit $D$ paid at the moment that the policyholder dies and a survival benefit $S$ paid at terminal time $T$ if the policyholder survives until then. The benefits $A, D$ and $S$ are time-dependent and contingent on the values of the risky assets $(Y, F)$.

We assume that the lifetimes of the policyholders $\left(\tau_{k}\right)_{k=1, \ldots, n}$ in the portfolio are independent and exponentially distributed, i.e.

$$
\mathbb{P}\left(\tau_{k}>t\right)=e^{-\lambda t}, \quad k=1, \ldots, n, \quad 0 \leq t \leq T .
$$

We also assume that $\left(\tau_{k}\right)_{k=1, \ldots, n}$ are independent of the financial market. For simplicity we use constant mortality intensity $\lambda$. Let

$$
N(t)=\sum_{k=1}^{n} \mathbf{1}\left\{\tau_{k} \leq t\right\}, \quad J(t)=n-N(t), \quad 0 \leq t \leq T,
$$

where $N(t)$ counts the number of deaths until time $t$, and $J(t)$ counts the number of in-force policies in the insurance portfolio at time $t$. We also introduce the compensated counting process

$$
\tilde{N}(t)=N(t)-\int_{0}^{t}(n-N(s-)) \lambda d s, \quad 0 \leq t \leq T,
$$

which we use to define a stochastic integral for the insurance risk.

The stream of benefits from the insurance portfolio is modelled by the process $B=$ $(B(t), 0 \leq t \leq T)$, which is described by the equation

$$
\begin{aligned}
B(t)= & \int_{0}^{t}(n-N(u-)) A(u, Y(u), F(u)) d u+\int_{0}^{t} D(u, Y(u), F(u)) d N(u) \\
& +(n-N(T)) S(Y(T), F(T)) \mathbf{1}_{t=T}, \quad 0 \leq t \leq T
\end{aligned}
$$

In our model the insurer is exposed to three sources of risk: 
- Tradeable (hedgeable) financial risk $Y$ : The fluctuations of the risky asset $Y$ impact the payment process 2.5 . This risk can be perfectly hedged by trading in $Y$.

- Non-tradeable (non-hedgeable) financial risk $F$ : The variations of the risky asset $F$ impact the benefit stream (2.5) as well. This risk can be partially hedged by trading in $Y$, since $Y$ and $F$ are correlated.

- Non-tradeable (non-hedgeable) insurance risk $N$ : The risk arises since the policyholders die at random times and death-related benefits have to be paid at unpredictable times. This risk cannot be hedged since it is assumed to be independent of the financial market.

In this paper we focus on two special cases of $(2.5)$ which are relevant for applications.

Example 2.1. Tradeable and non-tradeable financial risk - Terminal claims: The insurer is only exposed to a terminal benefit $S(Y(T), F(T))$ which is depending on the tradeable risky asset $Y(T)$ and the non-tradeable risky asset $F(T)$. We choose $A(t, y, f)=$ $D(t, y, f)=\lambda=0$ in (2.5). The benefit stream (2.5) takes the form

$$
B(t)=S(Y(T), F(T)) \mathbf{1}\{t=T\} .
$$

Such claims are common in financial mathematics as payoffs of European-type options on tradeable assets (e.g. exchange-traded stocks) or options on non-tradeable assets (e.g. electricity, temperature, etc.). In insurance, such payoffs are also considered by Pelsser (2010) as unit-linked contracts in which the non-tradeable asset $F$ represents an insurance process partially correlated to the stock $Y$.

In particular, we will focus on valuating a put option on the non-tradeable risky asset $F$ :

$$
S(Y(T), F(T))=\max \left\{S^{*}-F(T), 0\right\} .
$$

Example 2.2. Non-tradeable insurance risk and tradeable financial risk - Unitlinked contracts: Consider a portfolio consisting of $n$ unit-linked insurance contracts in which each policyholder pays a single premium $P(0)$ at time 0 . An initial fee $e P(0)$ is deducted from the premium to cover administrative costs, and the remaining amount $F(0)=$ $P(0)(1-e)$ is invested by the insurer in the risky asset $Y$. The premium invested together with investment returns constitutes the so-called policyholder's fund. The policyholder's fund is treated as a non-tradeable risky asset $F$ and its value is modelled with 2.3 . However, the insurer charges fees from the policyholder's fund. Hence, we define the following dynamics for the non-tradeable risky asset $F$ :

$$
\frac{d F(t)}{F(t)}=\left(\mu_{Y}-c\right) d t+\sigma_{Y} d W_{Y}(t)
$$


where $c$ denotes the continuously deducted fee from the policyholder's fund to be paid for the guarantees stipulated in the unit-linked contract. We point out that the correlation coefficient $\rho$ between $W_{F}$ and $W_{Y}$ is here equal to 1 ( since $W_{F}=W_{Y}$ ).

The unit-linked contracts offer death and survival benefits. The policyholders own their funds and the insurer guarantees a minimal death benefit $D^{*}$ (also called GMDB) and a minimal survival benefit $S^{*}$ (also called GMMB). For each policy, the insurer is exposed to the death and survival guarantees: $\max \left\{D^{*}-F(t), 0\right\}$ and $\max \left\{S^{*}-F(T), 0\right\}$. Hence in the benefit stream 2.5 we choose

$$
\begin{aligned}
D(t, Y(t), F(t)) & =\max \left\{D^{*}-F(t), 0\right\} \\
S(Y(T), F(T)) & =\max \left\{S^{*}-F(T), 0\right\} .
\end{aligned}
$$

Since the insurer collects the fees from the policyholders' funds continuously, in (2.5) we must set

$$
A(t, Y(t), F(t))=-c F(t)
$$

The benefit stream 2.5 in this example is then given by

$$
\begin{aligned}
B(t)= & -\int_{0}^{t}(n-N(u-)) c F(u) d u+\int_{0}^{t}\left(D^{*}-F(u)\right)^{+} d N(u) \\
& +(n-N(T))\left(S^{*}-F(T)\right)^{+} \mathbf{1}_{t=T},
\end{aligned}
$$

where $\left(x-x^{*}\right)^{+}=\max \left\{x-x^{*}, 0\right\}$. Even though the guarantees and the fees are contingent on the non-tradeable risky asset $F$, they can be perfectly replicated by dynamic trading in the financial market since the risky asset $F$ is perfectly correlated with the tradeable risky asset $Y$. Consequently, the claims from the benefit stream (2.9) are contingent on the tradeable financial risk and the non-tradeable insurance risk.

\subsection{Arbitrage-free pricing}

From arbitrage-free pricing theory, it follows that the price at time $t$ of the process $B$ can be expressed as

$$
\varphi_{B}(t)=\mathbb{E}^{\mathbb{Q}}\left[\int_{t}^{T} e^{-r(s-t)} d B(s) \mid \mathcal{F}_{t}\right], \quad 0 \leq t \leq T,
$$

with an equivalent martingale measure $\mathbb{Q}$. We now describe the set of equivalent martingale measures in our combined financial and insurance model. Let $\mathbb{P}$ denote the real-world measure under which the dynamics $(2.2), 2.3)$ and $(2.5)$ are specified. We define

$$
\begin{aligned}
\frac{d \mathbb{Q}}{d \mathbb{P}} \mid \mathcal{F}_{t} & =\mathcal{M}^{\zeta, \chi}(t), \quad 0 \leq t \leq T, \\
\frac{d \mathcal{M}^{\zeta, \chi}(t)}{\mathcal{M}^{\zeta, \chi}(t-)} & =-\left(\frac{\mu_{Y}-r}{\sigma_{Y}}\right) d W_{1}(t)+\zeta(t) d W_{2}(t)+\chi(t) d \tilde{N}(t),
\end{aligned}
$$


for some processes $(\zeta, \chi)$. By Girsanov's theorem we deduce that

$$
\begin{aligned}
& W_{1}^{\mathbb{Q}}(t)=W_{1}(t)+\int_{0}^{t}\left(\frac{\mu_{Y}-r}{\sigma_{Y}}\right) d s, \quad 0 \leq t \leq T, \\
& W_{2}^{\mathbb{Q}}(t)=W_{2}(t)-\int_{0}^{t} \zeta(s) d s, \quad 0 \leq t \leq T, \\
& \tilde{N}^{\mathbb{Q}}(t)=N(t)-\int_{0}^{t}(n-N(s-)) \lambda(s)(1+\chi(s)) d s, \quad 0 \leq t \leq T,
\end{aligned}
$$

are $\mathbb{Q}$-Brownian motions and $\mathbb{Q}$-compensated counting process. The processes $(\zeta, \chi)$ are called the risk premiums for the non-tradeable financial and insurance risk, and $\frac{\mu_{Y}-r}{\sigma_{Y}}$ is the risk premium for the tradeable financial risk. We will show that our valuation operator implies particular values for $(\zeta, \chi)$.

Finally, we will consider a special case for $\mathbb{Q}$. We define

$$
\begin{aligned}
\frac{d \hat{\mathbb{Q}}}{d \mathbb{P}} \mid \mathcal{F}_{t} & =\hat{\mathcal{M}}(t), \quad 0 \leq t \leq T, \\
\frac{d \hat{\mathcal{M}}(t)}{\hat{\mathcal{M}}(t)} & =-\left(\frac{\mu_{Y}-r}{\sigma_{Y}}\right) d W_{1}(t) .
\end{aligned}
$$

The measure $\hat{\mathbb{Q}}$ can be considered as the most objective measure from the set of subjective measures $\mathbb{Q}$ from (2.11) since no subjective assumptions on $(\zeta, \chi)$ are made to define $\hat{\mathbb{Q}}$. Equivalently stated, there are zero risk premiums for the non-tradeable risks and their dynamics under $\hat{\mathbb{Q}}$ and $\mathbb{P}$ coincide.

\section{The one-period valuation operator}

In this section we present our one-period valuation operator which is the building block for the multi-period, discrete-time valuation operator, and for the continuous-time valuation operator.

We split the valuation of the benefit stream $B$ into two parts and we valuate a hedgeable and a non-hedgeable part of $B$ separately. We define the value of a hedgeable part of the benefit stream (2.5) as the initial market costs of a hedging portfolio for the benefit stream. Let $\theta=(\theta(t), 0 \leq t \leq T)$ denote the amount of money invested in the risky asset $Y$ and $V^{\theta}=\left(V^{\theta}(t), 0 \leq t \leq T\right)$ the hedging portfolio under the strategy $\theta$. The dynamics of the hedging portfolio $V^{\theta}$ is described with the following SDE:

$$
\begin{aligned}
d V^{\theta}(t)= & \theta(t)\left(\mu_{Y} d t+\sigma_{Y} d W_{Y}(t)\right)+\left(V^{\theta}(t)-\theta(t)\right) r d t \\
& -(n-N(t-)) A(t, Y(t), F(t)) d t-D(t, Y(t), F(t)) d N(t), \quad 0 \leq t \leq T,
\end{aligned}
$$


and the terminal claims $(n-N(T)) S(Y(T), F(T))$ are subtracted from $V^{\theta}(T)$ at the terminal time $T$. We determine the optimal dynamic hedging strategy and the optimal hedging portfolio by quadratic hedging under the equivalent martingale measure $\hat{\mathbb{Q}}$, i.e. we solve:

$$
\begin{aligned}
& \inf _{\theta} \mathbb{E}^{\hat{\mathbb{Q}}}\left[\left|(n-N(T)) S(Y(T), F(T))-V^{\theta}(T)\right|^{2}\right], \\
& V^{\theta} \text { satisfies the dynamics } 3.1 .
\end{aligned}
$$

The solution to the quadratic hedging problem (3.2) can be found in Proposition 3.1 in Delong et al. (2018). The initial value of the optimal hedging portfolio and the optimal dynamic hedging strategy are given by

$$
\begin{gathered}
V_{B}^{*}(0)=v^{n}\left(0, y_{0}, f_{0}\right), \\
\theta_{B}^{*}(t)=v_{y}^{J(t-)}(t, Y(t), F(t)) Y(t)+v_{f}^{J(t-)}(t, Y(t), F(t)) F(t) \frac{\sigma_{F}}{\sigma_{Y}} \rho, \quad 0 \leq t \leq T,
\end{gathered}
$$

where

$v^{k}(t, y, f)=\mathbb{E}_{t, y, f, k}^{\hat{\mathbb{Q}}}\left[\int_{t}^{T} e^{-r(u-t)} d B(u)\right], \quad(t, y, f) \in[0, T] \times(0, \infty) \times(0, \infty), k \in\{0, \ldots, n\}$.

The next examples confirm that the initial value of the optimal hedging portfolio $V_{B}^{*}$ can be used to define the value of the hedgeable part of the benefit stream $B$.

Example 3.1. Let us consider the benefit stream from Example 2.1 with the terminal benefit $S(Y(T), F(T))=\left(S^{*}-F(T)\right)^{+}$. From the dynamics of the risky asset (2.3) and the change of measure (2.12), we can deduce that

$$
F(t)=e^{\delta_{F}^{\hat{\varphi}} t} \tilde{F}(t)
$$

where $\delta_{F}^{\hat{\mathbb{Q}}}=\mu_{F}-\frac{\mu_{Y}-r}{\sigma_{Y}} \sigma_{F} \rho-r$ is the excess return of the risky asset $F$ over the risk-free rate under $\hat{\mathbb{Q}}$, and

$$
\frac{d \tilde{F}(t)}{\tilde{F}(t)}=r d t+\sigma_{F} d W_{F}^{\hat{\mathbb{Q}}}(t)
$$

We directly find that

$$
\begin{aligned}
v^{k}(t, y, f) & =v(t, f)=\mathbb{E}_{t, f}^{\hat{\mathbb{Q}}}\left[e^{-r(T-t)}\left(S^{*}-e^{\delta_{F}^{\hat{\mathbb{Q}}}(T-t)} \tilde{F}(T)\right)^{+}\right] \\
& =e^{\delta_{F}^{\hat{\mathbb{Q}}}(T-t)} P\left(t, T, f, \sigma_{F}, S^{*} e^{-\delta_{F}^{\hat{\mathbb{Q}}}(T-t)}\right),
\end{aligned}
$$

where $P(t, T, f, \sigma, X)$ denotes the value of the put option at time $t$ with maturity $T$ and strike $X$ in the Black-Scholes model with volatility $\sigma$ when the underlying is equal to $f$ at 
time $t$. By (3.3)-(3.4), the initial value of the optimal hedging portfolio and the optimal dynamic hedging strategy are given by

$$
\begin{aligned}
V_{B}^{*}(0) & =e^{\delta_{F}^{\hat{Q}} T} P\left(0, T, f_{0}, \sigma_{F}, S^{*} e^{-\delta_{F}^{\hat{Q}} T}\right) \\
\theta_{B}^{*}(t) & =e^{\delta_{F}^{\hat{Q}}(T-t)} P_{f}\left(t, T, F(t), \sigma_{F}, S^{*} e^{\delta_{F}^{\hat{Q}}(T-t)}\right) F(t) \frac{\sigma_{F}}{\sigma_{Y}} \rho .
\end{aligned}
$$

The strategy (3.7) is a delta-hedging strategy for the tradeable component of the risky asset $F$, which is correlated with the tradeable risky asset $Y$. The value $[3.6)$ is the market cost of such a hedging strategy in the financial market consisting of $(R, Y, F)$ with zero risk premium for the non-tradeable component of the risky asset $F$, which is independent of $Y$.

Example 3.2. Let us consider the benefit stream (2.9) from the unit-linked portfolio introduced in Example 2.2. First, we determine the conditional expectation under $\hat{\mathbb{Q}}$ of the terminal claims. By the independence between $F$ and $N$ which holds under $\hat{\mathbb{Q}}$ and the independence of the policyholders' lifetimes under $\hat{\mathbb{Q}}$, we obtain

$$
\begin{aligned}
& \mathbb{E}_{t, f, k}^{\hat{\mathbb{Q}}}\left[e^{-r(T-t)}(n-N(T))\left(S^{*}-F(T)\right)^{+}\right] \\
& \quad=\mathbb{E}_{t, k}^{\hat{\mathbb{Q}}}[n-N(T)] \mathbb{E}_{t, f}^{\hat{\mathbb{Q}}}\left[e^{-r(T-t)}\left(S^{*}-e^{-c(T-t)} \tilde{F}(T)\right)^{+}\right] \\
& \quad=k e^{-\lambda(T-t)} e^{-c(T-t)} P\left(t, T, f, \sigma_{Y}, S^{*} e^{c(T-t)}\right),
\end{aligned}
$$

where

$$
\frac{d \tilde{F}(t)}{\tilde{F}(t)}=r d t+\sigma_{Y} d W_{Y}^{\hat{\mathbb{Q}}}(t)=\frac{d Y(t)}{Y(t)} .
$$

Therefore, the function $v^{k}$ for the benefit stream 2.9 is given by

$$
\begin{aligned}
v^{k}(t, y, f)= & v^{k}(t, f)=k \int_{t}^{T} e^{-c(s-t)} P\left(t, s, f, \sigma_{Y}, D^{*} e^{c(s-t)}\right) \lambda e^{-\lambda(s-t)} d s \\
& +k e^{-c(T-t)} P\left(t, T, f, \sigma_{Y}, S^{*} e^{c(T-t)}\right) e^{-\lambda(T-t)}-k c f \int_{t}^{T} e^{-c(s-t)} e^{-\lambda(s-t)} d s .
\end{aligned}
$$

The initial value of the optimal hedging portfolio and the optimal dynamic hedging strategy are given by

$$
\begin{aligned}
V_{B}^{*}(0)= & v^{n}\left(0, f_{0}\right) \\
\theta_{B}^{*}(t)= & (n-N(t-)) F(t)\left(\int_{t}^{T} e^{-c(s-t)} P_{f}\left(t, s, f, \sigma_{Y}, D^{*} e^{c(s-t)}\right) \lambda e^{-\lambda(s-t)} d s\right. \\
& \left.+e^{-c(T-t)} P_{f}\left(t, T, f, \sigma_{Y}, S^{*} e^{c(T-t)}\right) e^{-\lambda(T-t)}-c \int_{t}^{T} e^{-c(s-t)} e^{-\lambda(s-t)} d s\right) .
\end{aligned}
$$

Under the hedging strategy $(3.10)$ the insurer can perfectly replicate the guarantees embedded in the benefit stream 2.9 if the timing of the cash-flows from the guarantees agrees 
with the expected run-off of the insurance portfolio. The value 3.9 is the market cost of such a hedging strategy in the complete financial market consisting of $(R, Y)$ with deterministic times of cash-flows. The hedging strategy 3.10$)$ is often applied in practice as the insurers often assume that the insurance risk is completely diversified, hence deterministic, and construct hedges for the financial risk.

Applying the hedging strategy (3.4) we can hedge the hedgeable part of the benefit stream $B$ and we are left with a non-hedgeable part of the benefit stream $B$. The non-hedgeable part of the benefit stream 2.5 is valuated with a subjective actuarial valuation operator $\pi$. Consequently, we propose to use the following one-period valuation operator $\varrho$ :

$$
\varrho(B)=V_{B}^{*}(0)+\pi\left(\left((n-N(T)) S(Y(T), F(T))-V_{B}^{*}(T)\right) e^{-r T}\right)
$$

where $V_{B}^{*}(t)$ denotes the value of the optimal hedging portfolio from (3.3)- $(3.4)$, and $\pi$ denotes a one-period actuarial valuation operator. In line with traditional actuarial principles, we decompose the actuarial valuation operator $\pi$ into the expected value operator and the actuarial risk margin, i.e. we set

$$
\pi(\xi)=\mathbb{E}^{\mathbb{P}}[\xi]+R M[\xi]
$$

Our one-period valuation operator (3.11) takes the form

$$
\begin{aligned}
\varrho(B)= & V_{B}^{*}(0)+\mathbb{E}^{\mathbb{P}}\left[\left((n-N(T)) S(Y(T), F(T))-V_{B}^{*}(T)\right) e^{-r T}\right] \\
& +R M\left[\left((n-N(T)) S(Y(T), F(T))-V_{B}^{*}(T)\right) e^{-r T}\right] .
\end{aligned}
$$

In this paper, we focus on the standard deviation as the actuarial risk margin:

$$
R M(\xi)=\frac{1}{2} \gamma \sqrt{\operatorname{Var}^{\mathbb{P}}[\xi]}
$$

where $\gamma$ denotes the risk aversion parameter of the insurer.

Let us briefly discuss the price given by the one-period valuation operator 3.12 . The first two terms in 3.12 can be interpreted as the best estimate of the future claims generated by the hedgeable and non-hedgeable risks of the benefit stream $B$, respectively. The initial value of the hedging portfolio $V_{B}^{*}(0)$ gives us the market cost (under zero risk premiums for the non-tradeable risks) of the investment strategy which replicates the hedgeable part of the liability $B$. The expected value operator provides the expected cost, under the real-world measure $\mathbb{P}$, of the non-hedgeable claims which remain after the application of the hedging portfolio $V_{B}^{*}$. The third term in 3.12 yields an additional capital which is used by the insurer to cover the non-hedgeable part of the liability $B$ in adverse scenarios when the capital determined by the first two terms is not sufficient to pay the claims. In the next 
section we will show that this desired decomposition of the price of the liability also holds for the continuous-time valuation operator.

Pelsser (2010) and Pelsser \& Ghalehjooghi (2016) propose a completely different oneperiod valuation operator $\varrho$, called a two-step valuation operator. Dhaene et al. (2017) and Barigou \& Dhaene (2018) use the one-period valuation operator (3.11) but they consider static hedging strategies. Let us compare our hedging strategy (3.4) and the valuation operator (3.12) with the hedging strategies and the valuation operators from the papers mentioned.

Example 3.3: We consider the benefit stream

$$
B(t)=(n-N(T)) Y(T) \mathbf{1}\{t=T\},
$$

which is a special case of the benefit stream from Example 2.2. All policyholders still alive at time $T$ get one unit of the risky asset $Y$. For simplicity of comparison, we assume that the returns of $(R, Y)$ are set equal to zero: $\mu_{Y}=r=0$. We note that the assumption implies that the risky asset $Y$ is a $\mathbb{P}$ and $\hat{\mathbb{Q}}$-martingale. Let the terminal benefit $(n-N(T)) Y(T)$ be denoted by $\triangle B(T)$.

Pelsser (2010) and Pelsser \& Ghalehjooghi (2016) suggest a two-step valuation operator to quantify the risk in a combined financial and insurance model in one-period. Simply put, the first step consists of determining the actuarial value conditional on the financial market evolution. In the second step, the actuarial value from the first step is treated as a tradeable claim in a complete financial market and its value is calculated (or in other words, the financial risk is quantified) with the expectation operator under the unique equivalent martingale measure $\hat{\mathbb{Q}}$. If we apply their valuation operator, we end up with the price

$$
\begin{aligned}
\varrho_{1}(B) & =\mathbb{E}^{\hat{\mathbb{Q}}}\left[\mathbb{E}^{\mathbb{P}}[\triangle B(T) \mid Y(T)]+\frac{1}{2} \gamma \sqrt{\operatorname{Var}^{\mathbb{P}}[\triangle B(T) \mid Y(T)}\right] \\
& =n p(0, T) Y(0)+\frac{1}{2} \gamma \sqrt{n p(0, T)(1-p(0, T))} Y(0),
\end{aligned}
$$

where we introduce the survival probabilities $p(s, t)=e^{-\lambda(t-s)}$. The valuation operator from Pelsser (2010) and Pelsser \& Ghalehjooghi (2016) does not provide any hedging strategy.

Dhaene et al. (2017) and Barigou \& Dhaene (2018) suggest to apply the valuation operator (3.11) and find the static hedging portfolio by solving the quadratic hedging problem (3.2) under the real-world measure $\mathbb{P}$. From Theorem 1 from Barigou \& Dhaene (2018), the static hedging strategy and the value of the hedging portfolio are given by

$$
\theta_{B}^{*}(0)=n p(0, T) Y(0), \quad V_{B}^{*}(t)=n p(0, T) Y(t)
$$


Therefore, the value of the benefit stream $B$, determined by (3.11), is equal to

$$
\begin{aligned}
\varrho_{2}(B) & \left.=V_{B}^{*}(0)+\mathbb{E}^{\mathbb{P}}\left[\triangle B(T)-V_{B}^{*}(T)\right]+\frac{1}{2} \gamma \sqrt{\operatorname{Var}^{\mathbb{P}}\left[\triangle B(T)-V_{B}^{*}(T)\right.}\right] \\
& =n p(0, T) Y(0)+\frac{1}{2} \gamma \sqrt{n p(0, T)(1-p(0, T)) \mathbb{E}^{\mathbb{P}}\left[\left.Y(T)\right|^{2}\right]} .
\end{aligned}
$$

Finally, we apply the valuation operator (3.11) but we use the dynamic hedging strategy found by solving (3.2). By Example 3.2, we find that the dynamic hedging strategy and the initial value of the hedging portfolio are given by

$$
\theta_{B}^{*}(t)=(n-N(t-)) p(t, T) Y(t), \quad V_{B}^{*}(0)=n p(0, T) Y(0) .
$$

From the predictable representation of the martingale $\mathbb{E}^{\mathbb{P}}\left[\triangle B(T) \mid \mathcal{F}_{t}\right]=(n-N(t)) p(t, T) Y(t)$, see e.g. Proposition 8.1.1 in Delong (2013), we can conclude that

$$
\begin{aligned}
\triangle B(T) & =\mathbb{E}^{\mathbb{P}}[\triangle B(T)]+\int_{0}^{T}(n-N(s-)) p(s, T) Y(s) \sigma d W^{\mathbb{P}}(s)-\int_{0}^{T} p(s, T) Y(s) d \tilde{N}^{\mathbb{P}}(s) \\
& =V_{B}^{*}(T)-\int_{0}^{T} p(s, T) Y(s) d \tilde{N}^{\mathbb{P}}(s),
\end{aligned}
$$

where the last line is deduced from the optimal hedging strategy $\theta_{B}^{*}$ and the dynamics of the hedging portfolio (3.1). Since the stochastic integrals involved are martingales, we immediately find that

$$
\mathbb{E}^{\mathbb{P}}\left[\triangle B(T)-V_{B}^{*}(T)\right]=0 .
$$

By the second moment property of the stochastic integrals, we can also calculate

$$
\begin{aligned}
& \operatorname{Var}^{\mathbb{P}}\left[\triangle B(T)-V_{B}^{*}(T)\right]=\mathbb{E}^{\mathbb{P}}\left[\left|\int_{0}^{T} p(s, T) Y(s) d \tilde{N}^{\mathbb{P}}(s)\right|^{2}\right] \\
& \quad=\mathbb{E}^{\mathbb{P}}\left[\int_{0}^{T} p^{2}(s, T) Y^{2}(s)(n-N(s)) \lambda d s\right]=n p(0, T) \mathbb{E}^{\mathbb{P}}\left[\int_{0}^{T} p(s, T) Y^{2}(s) \lambda d s\right],
\end{aligned}
$$

where we used the property that $p(0, s) p(s, T)=p(0, T)$ and the independence between $N$ and $Y$. In consequence, the value of the benefit stream $B$, determined by (3.11), is equal to

$$
\begin{aligned}
\varrho_{3}(B)= & n p(0, T) Y(0) \\
& +\frac{1}{2} \gamma \sqrt{n p(0, T)(1-p(0, T))} \sqrt{\int_{0}^{T} \mathbb{E}^{\mathbb{P}}\left[|Y(s)|^{2}\right] \frac{e^{\lambda s}}{e^{\lambda T}-1} \lambda d s .}
\end{aligned}
$$

Since $\mathbb{E}\left[|Y(s)|^{2}\right]>|E[Y(s)]|^{2}=Y^{2}(0)$ and $\mathbb{E}\left[|Y(s)|^{2}\right]=Y(0)^{2} e^{\sigma^{2} s}<\mathbb{E}\left[|Y(T)|^{2}\right]$, for all $s \in[0, T]$, we obtain the following order between the three types of valuations:

$$
\varrho_{1}(B)<\varrho_{3}(B)<\varrho_{2}(B) .
$$


It is clear that our valuation operator yields a lower price than the operator from Dhaene et al. (2017) and Barigou \& Dhaene (2018) since we allow for dynamic hedging in continuous time and the hedging strategy is updated with the current information on the insurance risk (the number of survivors). As expected, the one-period valuation operator suggested by Pelsser (2010) and Pelsser \& Ghalehjooghi (2016) is different from ours. Interestingly, in the next section we show that these two operators coincide when we consider the continuous-time limit of the discrete-time operator with a time step $h \rightarrow 0$.

\section{The continuous-time valuation operator}

The price at time $t$ of the future claims from the process $B$ is denoted by $\varphi_{B}(t)$. Let $\mathcal{T}=\{0, h, \ldots, T-h, T\}$ for a fixed time step $h$. The price $\varphi_{B}(t)$ at $t \in \mathcal{T}$ is defined by the operator

$$
\begin{aligned}
\varphi_{B}(T)= & (n-N(T)) S(Y(T), F(T)), \\
\varphi_{B}(t)= & \varrho_{t}\left(\int_{t}^{t+h} d \tilde{B}(s)\right), \quad t=0, h, \ldots, T-h, \\
\tilde{B}(s)= & \int_{t}^{s}(n-N(u-)) A(u, Y(u), F(u)) d u+\int_{t}^{s} D(u, Y(u), F(u)) d N(u) \\
& +\varphi_{B}(t+h) \mathbf{1}\{s=t+h\}, \quad t \leq s \leq t+h,
\end{aligned}
$$

where the operator $\varrho_{t}$ is the conditional version of (3.12), based on the up-to-date information on the financial and insurance risks available at time $t$. We point out that $\tilde{B}$ in $(4.1)$ is similar to $B$ in (2.5), and the benefit streams only differ with respect to the terminal claims. In a multi-period model, in each subperiod $[t, t+h]$ the insurer must optimally hedge the claims $A, D$ arriving from the benefit stream $B$ and the value of $B$ at the terminal time $t+h$.

We now extend the definition of the price $\varphi_{B}(t)$ from $t \in \mathcal{T}$ to all times $t \in[0, T]$. From (3.12) and 4.1) we can deduce that the discrete-time valuation operator $\varphi=\left(\varphi_{B}(t)\right)_{t \in \mathcal{T}}$ satisfies the discrete-time pricing equation:

$$
\begin{aligned}
\mathbb{E}^{\mathbb{P}} & {\left[\left(\varphi(t+h)-V_{\tilde{B}}^{*}(t+h)\right) e^{-r h}-\left(\varphi(t)-V_{\tilde{B}}^{*}(t)\right) \mid \mathcal{F}_{t}\right] } \\
& \left.\quad+R M\left[\left(\varphi(t+h)-V_{\tilde{B}}^{*}(t+h)\right)\right) e^{-r h}-\left(\varphi(t)-V_{\tilde{B}}^{*}(t)\right) \mid \mathcal{F}_{t}\right]=0, \quad t=0, h, \ldots, T-h,
\end{aligned}
$$

where the hedging portfolio $V_{\widetilde{B}}^{*}$ is derived from the mean-square hedging objective 3.2 with the benefit stream $\tilde{B}$, and the actuarial risk margin $R M$ is chosen by the insurer. The continuous-time valuation operator $\varphi=\left(\varphi_{B}(t)\right)_{t \in[0, T]}$ is defined as an operator which satisfies the continuous-time limit of the discrete-time pricing equation $(4.2)$ as the time step $h \rightarrow 0$. 
More precisely, we are interested in finding an operator $\varphi$ which satisfies

$$
\begin{gathered}
\lim _{h \rightarrow 0}\left\{\frac{\mathbb{E}_{t, y, f, k}\left[\left(\varphi(t+h)-V_{\tilde{B}}^{*}(t+h)\right) e^{-r h}-\left(\varphi(t)-V_{\tilde{B}}^{*}(t)\right)\right]}{h}\right. \\
\left.\quad+\frac{R M_{t, y, f, k}\left[\left(\varphi(t+h)-V_{\tilde{B}}^{*}(t+h)\right) e^{-r h}-\left(\varphi(t)-V_{\tilde{B}}^{*}(t)\right)\right]}{h}\right\}=0,
\end{gathered}
$$

for any $(t, y, f, k) \in[0, T) \times(0, \infty) \times(0, \infty) \times\{0, \ldots, n\}$.

In Theorem 5.1 in Delong et al. (2018) we derived the continuous-time limit of the discrete-time pricing equation (4.3) as well as the dynamic hedging strategy which underlies the continuous-time valuation operator $\varphi$. Let us choose standard deviation (3.13) scaled with $\sqrt{h}$ as the one-period actuarial risk margin $R M$. The continuous-time valuation operator $\varphi$ satisfies the system of PDEs:

$$
\begin{aligned}
& \varphi_{t}^{k}(t, y, f)+\varphi_{y}^{k}(t, y, f) y r+\varphi_{f}^{k}(t, y, f) f\left(\mu_{F}-\frac{\mu_{Y}-r}{\sigma_{Y}} \sigma_{F} \rho\right) \\
& +\varphi_{y f}^{k}(t, y, f) y f \sigma_{Y} \sigma_{F} \rho+\frac{1}{2} \varphi_{y y}^{k}(t, y, f) y^{2} \sigma_{Y}^{2}+\frac{1}{2} \varphi_{f f}^{k}(t, y, f) f^{2} \sigma_{F}^{2} \\
& +k A(t, y, f)+\left(\varphi^{k-1}(t, y, f)+D(t, y, f)-\varphi^{k}(t, y, f)\right) k \lambda-\varphi^{k}(t, y, f) r \\
& +\Phi^{k}\left(\varphi_{f}^{k}(t, y, f) f \sigma_{F} \sqrt{1-\rho^{2}}, \varphi^{k-1}(t, y, f)+D(t, y, f)-\varphi^{k}(t, y, f)\right)=0, \\
& \quad(t, y, f) \in[0, T) \times(0, \infty) \times(0, \infty), \\
& \varphi^{k}(T, y, f)=k S(y, f), \quad(y, f) \in(0, \infty) \times(0, \infty),
\end{aligned}
$$

for $k \in\{0, \ldots, n\}$, where $\Phi^{k}\left(x_{1}, x_{2}\right)=\frac{1}{2} \gamma \sqrt{x_{1}^{2}+x_{2}^{2} k \lambda}$, and

$$
\begin{array}{r}
\Phi^{k}\left(\varphi_{f}^{k}(t, y, f) f \sigma_{F} \sqrt{1-\rho^{2}}, \varphi^{k-1}(t, y, f)+D(t, y, f)-\varphi^{k}(t, y, f)\right) \\
\quad=\lim _{h \rightarrow 0} \frac{R M_{t, y, f, k}\left[\left(\varphi(t+h)-V_{\tilde{B}}^{*}(t+h)\right) e^{-r h}-\left(\varphi(t)-V_{\tilde{B}}^{*}(t)\right)\right]}{h} .
\end{array}
$$

By (3.12) and 4.5), the function $\Phi$, which we have in the PDEs 4.4), can be called an instantaneous actuarial risk margin and, intuitively, it puts a price on the non-hedgeable financial and insurance risks in the continuous-time model. The hedging strategy which underlies the continuous-time valuation operator $\varphi$ is given by

$$
\vartheta^{*}(t)=\varphi_{y}^{J(t-)}(t, Y(t), F(t)) Y(t)+\varphi_{f}^{J(t-)}(t, Y(t), F(t)) F(t) \frac{\sigma_{F}}{\sigma_{Y}} \rho, \quad 0 \leq t \leq T .
$$

We illustrate Theorem 5.1 from Delong et al. (2018) for the benefit streams from Examples 2.1-2.2. 
Example 4.1. Consider Example 2.1. The system of PDEs 4.4 reduces to one single PDE:

$$
\begin{aligned}
& \varphi_{t}(t, y, f)+\varphi_{y}(t, y, f) y r+\varphi_{f}(t, y, f) f\left(\mu_{F}-\frac{\mu_{Y}-r}{\sigma_{Y}} \sigma_{F} \rho\right) \\
& +\varphi_{y f}(t, y, f) y f \sigma_{Y} \sigma_{F} \rho+\frac{1}{2} \varphi_{y y}(t, y, f) y^{2} \sigma_{Y}^{2}+\frac{1}{2} \varphi_{f f}(t, y, f) f^{2} \sigma_{F}^{2}-\varphi(t, y, f) r \\
& +\Phi\left(\varphi_{f}(t, y, f) f \sigma_{F} \sqrt{1-\rho^{2}}\right)=0, \quad(t, y, f) \in[0, T) \times(0, \infty) \times(0, \infty), \\
& \varphi(T, y, f)=S(y, f), \quad(y, f) \in(0, \infty) \times(0, \infty),
\end{aligned}
$$

where $\Phi(x)=\frac{1}{2} \gamma|x|$. The insurer is exposed to the hedgeable and the non-hedgeable financial risk. The instantaneous actuarial risk margin $\Phi$ puts a price on the non-hedgeable financial risk, which is related to the independent component of the risky asset $F$.

The PDE (4.7) agrees with the PDE derived by Pelsser (2010) and Pelsser \& Ghalehjooghi (2016). In the previous section in Example 3.3, we demonstrated that the two-step valuation operator from Pelsser (2010) and Pelsser \& Ghalehjooghi (2016) is different from our valuation operator 3.12 if we consider a one-period approach. However, if we consider the continuous time limit of these operators, both valuation approaches coincide.

Example 4.2. Consider Example 2.2. The system of PDEs 4.4 takes now the following form:

$$
\begin{aligned}
& \varphi_{t}^{k}(t, f)+\varphi_{f}^{k}(t, f) f(r-c)+\frac{1}{2} \varphi_{f f}^{k}(t, f) f^{2} \sigma_{Y}^{2}-k c f \\
& +\left(\varphi^{k-1}(t, f)+D(f)-\varphi^{k}(t, f)\right) k \lambda-\varphi^{k}(t, f) r \\
& +\Phi^{k}\left(\varphi^{k-1}(t, f)+D(f)-\varphi^{k}(t, f)\right)=0, \quad(t, f) \in[0, T) \times(0, \infty), \\
& \varphi^{k}(T, f)=k S(f), \quad f \in(0, \infty)
\end{aligned}
$$

for $k=0, \ldots, n$, where $\Phi^{k}(x)=\frac{1}{2} \gamma \sqrt{x^{2} k \lambda}$. The insurer is exposed to the hedgeable financial risk and the non-hedgeable insurance risk. The instantaneous actuarial risk margin $\Phi$ puts a price on the non-hedgeable insurance risk, which is related to the independent policyholders' death.

We remark that we make the assumption that the fee $c$ is already given (by a pricing department) and we are interested in the fair valuation of the future claims from the benefits stream 2.9. Alternatively, the fee $c$ could also be determined by calculating the fair value of $B$ at time $t=0$ and setting it to zero (this will be performed in Section 5).

Applying the Feynman-Kac formula, see Theorem 5.2 in Delong et al. (2018), we find that our continuous-time valuation operator (4.4) has the following probabilistic representation:

$$
\begin{aligned}
& \varphi^{k}(t, y, f)= \mathbb{E}_{t, y, f, k}^{\hat{\mathbb{Q}}}\left[\int_{t}^{T} e^{-r(s-t)} d B(s)+\int_{t}^{T} e^{-r(s-t)} \Phi(s) d s\right], \\
&(t, y, f) \in[0, T] \times(0, \infty) \times(0, \infty), k \in\{0, \ldots, k\},
\end{aligned}
$$


where $\Phi$ is a short notation for the instantaneous actuarial risk margin

$$
\begin{aligned}
\Phi^{J(s)} & \left(\varphi_{f}^{J(s)}(s, Y(s), F(s)) F(s) \sigma_{F} \sqrt{1-\rho^{2}},\right. \\
& \left.\varphi^{J(s)-1}(s, Y(s), F(s))+D(s, Y(s), F(s))-\varphi^{J(s)}(s, Y(s), F(s))\right) .
\end{aligned}
$$

The representation (4.9) states that our valuation operator $\varphi$ valuates liabilities as the best estimate of the liability plus the total actuarial risk margin for the liability:

$$
\begin{aligned}
\varphi_{B} & =\text { Fair Value of } B \\
& =\text { Best Estimate of } B+\text { Total Actuarial Risk Margin for } B .
\end{aligned}
$$

In our continuous-time model we identify that the best estimate of a liability is the expected value of the future claims from the liability, where the expected value is taken under the measure $\hat{\mathbb{Q}}$ given by 2.13 . It perfectly agrees with intuition that the best estimate assumptions for the non-tradeable financial and insurance risks should not include any risk premiums for these risks, see also Happ et al. (2015). The best estimate assumption for the tradeable financial risk coincides with the risk premium derived from the tradeable risky asset $Y$ and is equivalent with risk-neutral valuation in the complete financial market with $(R, Y)$. We can deduce, as for the multi-period valuation operator (3.12), that the best estimate of a liability contingent on the hedgeable and non-hedgeable financial and insurance risks consists of the market cost of the replicating portfolio for the hedgeable part of the benefit stream and the expected, real-world, cost of the non-hedgeable claims which remain after the application of the hedging portfolio.

Let us interpret the total actuarial risk margin. We introduce the net asset value process $N A V:=(N A V(t), 0 \leq t \leq T)$. We define

$$
N A V(t)=V^{*}(t)-\varphi(t), \quad 0 \leq t \leq T
$$

where $V^{*}$ denotes the hedging portfolio (3.1) under the hedging strategy $\vartheta^{*}$ from (4.6) and $\varphi$ is the continuous-time valuation operator from (4.4). The net asset value process is the difference between the assets and the liabilities. By Theorem 5.1 and the following results from Delong et al. (2018), we find the dynamics

$$
\begin{aligned}
d N A V(s)= & N A V(s) r d s+\Phi(s) d s \\
& -\varphi_{f}^{J(s-)}(s, Y(s), F(s)) F(s) \sigma_{F} \sqrt{1-\rho^{2}} d W_{2}(s) \\
& -\left(\varphi^{J(s-)-1}(s, Y(s), F(s))+D(s, Y(s), F(s))\right. \\
& \left.\quad-\varphi^{J(s-)}(s, Y(s), F(s))\right) d \tilde{N}(s), \quad 0 \leq s \leq T .
\end{aligned}
$$

Since we don't have a stochastic integral driven by $W_{1}$ in 4.10 , the tradeable financial risk is eliminated by applying the hedging strategy (4.6). The insurer is only exposed to the 
non-tradeable financial and insurance risks. The two stochastic integrals in 4.10 describe the evolution of the non-hedgeable claims $d B(t)$ and the non-hedgeable change in the value of the claims $d \varphi(t)$ :

- The first stochastic integral describes the risk that the value of the claims changes due to a change in the non-hedgeable, independent component of the risky asset $F$. The integrand is the delta-hedging replication strategy for the non-tradeable, independent component of the risky asset $F$.

- The second stochastic integral describes the risk that in the case of the non-hedgeable, independent event of the policyholder's death the insurer pays the death benefit and recalculates the value of the claims for the in-force policies. The integrand is the sum at risk to which the insurer is exposed in the event of the policyholder's death.

The integrands, described above, can be interpreted as measures of the non-hedgeable financial and the non-hedgeable insurance risk. We can observe that the instantaneous actuarial risk margin $\Phi$ is a function of two variables which are directly related to the integrands of the stochastic integrals in 4.10 . Consequently, the instantaneous actuarial risk margin function combines the measures of the non-hedgeable financial and the non-hedgeable insurance risk into one measure of the total non-hedgeable risk. The fair value of the benefit stream $B$ is next derived by solving the PDEs (4.4) with the instantaneous actuarial risk margin $\Phi$.

Our valuation model works in the following way. At each time $t \in[0, T)$, the insurer should hold an additional capital, determined by the instantaneous actuarial risk margin $\Phi(t)$, which protects the insurer against adverse scenarios in the evolution of the nonhedgeable financial and insurance risks in an infinitesimal period of time $d t$. We can see that the instantaneous actuarial risk margin $\Phi$ offsets the differentials of the stochastic integrals for the non-hedgeable risks in 4.10 . At time $t=0$ the expected (best estimate) cost of providing the capitals $\Phi$ during the duration of the insurance portfolio is equal to

$$
\mathbb{E}^{\hat{\mathbb{Q}}}\left[\int_{0}^{T} e^{-r s} \Phi(s) d s\right] .
$$

This expectation is called the total actuarial risk margin and is the second part of the technical provision (4.9), apart from the best estimate discussed before. The technical provision (the value of the benefit stream), the best estimate of the liability and the cost of financing the future instantaneous actuarial risk margins are recalculated over the duration of the contract. If the initial technical provision, financed with the premiums collected from the policyholders and invested with the optimal hedging strategy, is not sufficient to cover the claims, the best estimate and the instantaneous actuarial risk margin at later times, then the additional capital is provided by the shareholders. Since the total actuarial risk 
margin should protect the insurer only against adverse scenarios in the evolution of the non-hedgeable financial and insurance risks, we expect that the insurer (the shareholders) should earn, on average, the instantaneous actuarial risk margins as the time passes. The instantaneous actuarial risk margins are released from the technical provision (4.9) and, on average, they are not used to cover the losses since the realized loss on the hedgeable risk is always zero and the expected loss on the non-hedgeable risks is also zero, both under $\mathbb{P}$ and $\hat{\mathbb{Q}}$ (the expected value of the stochastic integrals in 4.10 is zero). We find that

$$
\mathbb{E}\left[N A V(t) e^{-r(t-s)} \mid \mathcal{F}_{s}\right]=N A V(s)+\mathbb{E}\left[\int_{s}^{t} e^{-r(u-s)} \Phi(u) d u \mid \mathcal{F}_{s}\right], \quad 0 \leq s \leq t \leq T .
$$

The second term in 4.11 can be interpreted as the expected dividend to the shareholders.

Let us investigate in more details the valuation of the claims from Examples 4.1-4.2.

Example 4.3. We continue Example 4.1. We first derive the arbitrage-free representation (2.10) for the valuation operator $\varphi$ which solves the PDE (4.7). The arbitrage-free representation allows us to identify the risk premium for the measure 2.11) used for pricing the non-hedgeable financial risk, which is implied by our subjective valuation operator.

Let $\tilde{\mathbb{Q}}$ denote the equivalent martingale measure defined by 2.11 with the risk premium

$$
\zeta(t)=\frac{1}{2} \gamma \operatorname{sgn}\left(\varphi_{f}(t, Y(t), F(t))\right) .
$$

By Girsanov's theorem, the process

$$
W_{2}^{\tilde{\mathbb{Q}}}(t)=W_{2}^{\hat{\mathbb{Q}}}(t)-\frac{1}{2} \gamma \int_{0}^{t} \operatorname{sgn}\left(\varphi_{f}(s, Y(s), F(s))\right) d s
$$

is a $\tilde{\mathbb{Q}}$-Brownian motion and the dynamics of $(Y, F)$ under $\tilde{\mathbb{Q}}$ are given by

$$
\begin{aligned}
\frac{d Y(t)}{Y(t)}= & r d t+\sigma_{Y} d W_{Y}^{\tilde{\mathbb{Q}}}(t) \\
\frac{d F(t)}{F(t)}= & \left(\mu_{F}-\frac{\mu_{Y}-r}{\sigma_{Y}} \sigma_{F} \rho\right. \\
& +\frac{1}{2} \gamma \sigma_{F} \sqrt{1-\rho^{2}} \operatorname{sgn}\left(\varphi_{f}(t, Y(t), F(t))\right) d t+\sigma_{F} d W_{F}^{\tilde{\mathbb{Q}}}(t) .
\end{aligned}
$$

We can rewrite the PDE (4.7) as

$$
\begin{aligned}
& \varphi_{t}(t, y, f)+\varphi_{y}(t, y, f) y r \\
& +\varphi_{f}(t, y, f) f\left(\mu_{F}-\frac{\mu_{Y}-r}{\sigma_{Y}} \sigma_{F} \rho+\frac{1}{2} \gamma \sigma_{F} \sqrt{1-\rho^{2}} \operatorname{sgn}\left(\varphi_{f}(t, y, f)\right)\right) \\
& +\varphi_{y f}(t, y, f) y f \sigma_{Y} \sigma_{F} \rho+\frac{1}{2} \varphi_{y y}(t, y, f) y^{2} \sigma_{Y}^{2}+\frac{1}{2} \varphi_{f f}(t, y, f) f^{2} \sigma_{F}^{2}-\varphi(t, y, f) r=0, \\
& \varphi(T, y, f)=S(y, f) .
\end{aligned}
$$


Applying the Feynman-Kac formula, we can show that the solution to the PDE (4.13), and also the solution to the PDE (4.7), has the representation

$$
\varphi(t, y, f)=\mathbb{E}_{t, y, f}^{\tilde{\mathbb{Q}}}\left[e^{-r(T-t)} S(Y(T), F(T))\right] .
$$

Under the valuation operator $\varphi$ determined by the $\operatorname{PDE}(4.7)$, we value the claim $S$ contingent on $(Y, F)$ with the expected value operator under the measure $\tilde{\mathbb{Q}}$ which distorts the dynamics of the non-tradeable risky asset $F$. This distortion of the real-world probability measure is based on the subjective risk aversion coefficient in the risk margin applied by the insurer. We note that the dynamics of the tradeable risky asset $Y$ is objective under the measure $\tilde{\mathbb{Q}}$, in the sense that the expected discounted future value of the risky asset $Y$ under $\tilde{\mathbb{Q}}$ is equal to the current price of $Y$ (the martingale condition). From 4.12 we observe that if the insurer is exposed to an increase in the price of the asset $F, \operatorname{sgn}\left(\varphi_{f}\right)>0$, then the process $\zeta$ increases the drift of $F$, if the insurer is exposed to a decrease in the price of the asset $F, \operatorname{sgn}\left(\varphi_{f}\right)<0$, then the process $\zeta$ decreases the drift of $F$. Such a mechanism of updating the parameters of the model used for pricing the claim $S$ contingent on the non-tradeable risky asset $F$ agrees with intuition. The sign of the derivative $\varphi_{f}$, which determines whether the insurer is better off by an increase or decrease in the price of the asset $F$, depends on the terminal payoff $S$ and its relation to $F$.

Let us focus on a put option contingent on the non-tradeable asset $F$, i.e. we choose $S(y, f)=\left(S^{*}-f\right)^{+}$. We expect that the sign of the derivative $\varphi_{f}$ is negative since the insurer is worse off by a decrease in the price of the asset $F$. Let us assume that $\operatorname{sgn}\left(\varphi_{f}(t, y, f)\right)=$ -1 . From 4.12 and (4.14) we conclude that

$$
\varphi(t, y, f)=\varphi(t, f)=\mathbb{E}_{t, f}^{\tilde{\mathbb{Q}}}\left[e^{-r(T-t)}\left(S^{*}-F(T)\right)^{+}\right],
$$

where

$$
\begin{aligned}
\frac{d F(t)}{F(t)} & =\left(\mu_{F}-\frac{\mu_{Y}-r}{\sigma_{Y}} \sigma_{F} \rho-\frac{1}{2} \gamma \sigma_{F} \sqrt{1-\rho^{2}}\right) d t+\sigma_{F} d W_{F}^{\tilde{\mathbb{Q}}}(t), \\
& =\left(\delta_{F}^{\tilde{\mathbb{Q}}}+r\right) d t+\sigma_{F} d W_{F}^{\tilde{\mathbb{Q}}}(t),
\end{aligned}
$$

and $\delta_{F}^{\tilde{Q}}$ denotes the excess return of the risky asset $F$ over the risk-free rate under the measure $\tilde{\mathbb{Q}}$. The expected value 4.15 can be easily calculated. As in Example 3.1, we can deduce that

$$
\varphi(t, f)=e^{\delta_{F}^{\tilde{\Phi}}(T-t)} P\left(t, T, f, \sigma_{F}, S^{*} e^{-\delta_{F}^{\tilde{Q}}(T-t)}\right),
$$

where $P(t, T, f, \sigma, X)$ denotes the value of a put option at time $t$ with maturity $T$ and strike $X$ in the Black-Scholes model with volatility $\sigma$ when the value of the underlying at time $t$ is equal to $f$. Now, we have to confirm that the sign of the derivative $\varphi_{f}$, for the function 
$\varphi$ defined in (4.17), is indeed negative. Derivatives are well-known for put option prices. Consequently, we have

$$
\varphi_{f}(t, f)=-e^{\delta_{F}^{\tilde{\mathbb{Q}}}(T-t)} \mathcal{N}\left(-\frac{\log f-\log S^{*}+\delta_{F}^{\tilde{\mathbb{Q}}}(T-t)+\left(r+\frac{\sigma_{F}^{2}}{2}\right)(T-t)}{\sigma_{F} \sqrt{T-t}}\right)<0,
$$

where $\mathcal{N}$ denotes the cumulative distribution function of the standard normal distribution. We conclude that the function (4.17) solves the PDE (4.7) with $S(y, f)=\left(S^{*}-f\right)^{+}$and gives the price of the put option on the non-tradeable risky asset $F$ in our model.

Now, we focus on the representation (4.9) of the fair price of liability as the sum of the best estimate of the liability and the total actuarial risk margin for the liability. We have the following decomposition of the fair price:

$$
\begin{aligned}
\varphi(t, f)= & \mathbb{E}_{t, f}^{\hat{\mathbb{Q}}}\left[e^{-r(T-t)}\left(S^{*}-F(T)\right)^{+}\right] \\
& -\mathbb{E}_{t, f}^{\hat{\mathbb{Q}}}\left[\frac{1}{2} \gamma \sigma_{F} \sqrt{1-\rho^{2}} \int_{t}^{T} e^{-r(s-t)} \varphi_{f}(s, F(s)) F(s) d s\right] .
\end{aligned}
$$

The best estimate of the liability, the first term in (4.18), is the expected value of the discounted claim from the put option, where the expected value is taken under the measure $\hat{\mathbb{Q}}$. Intuitively, the best estimate of the hedgeable part of the put option $\left(S^{*}-F(T)\right)^{+}$results from taking the expected value of the hedgeable claim under the equivalent martingale measure $\hat{\mathbb{Q}}$, which is the unique martingale measure in the complete financial market consisting of $(R, Y)$. This value coincides with the market cost of the investment portfolio which perfectly replicates the hedgeable part of the put option. The best estimate of the non-hedgeable part of the put option $\left(S^{*}-F(T)\right)^{+}$results from taking the expected value of the non-hedgeable claim under the real-world measure $\mathbb{P}$. This value is the expected, real-world, cost of the claim left after the application of the optimal hedging portfolio. The best estimate in (4.18) is equal to

$$
B E=e^{\delta_{F}^{\hat{Q}}(T-t)} P\left(t, T, f, \sigma_{F}, S^{*} e^{-\delta_{F}^{\hat{\oplus}}(T-t)}\right),
$$

where $\delta_{F}^{\hat{\mathbb{Q}}}=\mu_{F}-\frac{\mu_{Y}-r}{\sigma_{Y}} \sigma_{F} \rho-r$ is the excess return of $F$ over the risk-free rate under the measure $\hat{\mathbb{Q}}$.

In this example, the insurer is exposed to changes in the non-tradeable asset price $F$, which imply changes in the price of the put option. The change of the asset price $F$ is driven by two components: a component correlated with the tradeable asset $Y$ and an independent, non-tradeable component. By (4.6) the optimal hedging strategy consists of applying the delta-hedging strategy

$$
\vartheta^{*}(t)=\varphi_{f}(t, F(t)) F(t) \frac{\sigma_{F}}{\sigma_{Y}} \rho
$$


Consequently, the insurer perfectly hedges the changes in the price of the claim $\varphi$ resulting from the component of $F$ correlated with $Y$. However, the changes in the price of the claim $\varphi$ resulting from the independent component of $F$ cannot be hedged. Recalling 4.10), we derive the dynamics of the net asset value process:

$$
\begin{aligned}
d N A V(s)= & N A V(s) r d t-\frac{1}{2} \gamma \sigma_{F} \sqrt{1-\rho^{2}} \varphi_{f}(s, F(s)) F(s) d s \\
& -\varphi_{f}(s, F(s)) F(s) \sigma_{F} \sqrt{1-\rho^{2}} d W_{2}(s) .
\end{aligned}
$$

The stochastic integral in 4.20 models the changes in the price of the put option resulting from changes in the non-tradeable asset $F$ induced by the component independent of the tradeable asset $Y$. At each time $t \in[0, T)$, the insurer must hold an additional capital in the amount of

$$
\Phi(t)=-\frac{1}{2} \gamma \sigma_{F} \sqrt{1-\rho^{2}} \varphi_{f}(t, F(t)) F(t),
$$

to compensate this non-hedgeable risk. The total actuarial risk margin, the second term in (4.18), is the expected total cost of providing the capitals which correspond in value with the delta-hedging strategy for the independent component of $F$. This delta-hedging strategy cannot be applied by the insurer since the risky asset $F$ is not traded. However, the necessary capitals to perform this delta-hedging strategy, are sufficient to cover the nonhedgeable financial loss (in our model where the insurer uses a standard deviation risk margin for quantifying the non-hedgeable risks).

From (4.16) we can conclude that the drift of the risky asset $F$ under the pricing measure $\tilde{\mathbb{Q}}$ is a decreasing function of the risk aversion coefficient $\gamma$, and from 4.15 , that the price $\varphi$ is an increasing function of the risk aversion coefficient $\gamma$. Consequently, the higher the risk aversion coefficient of the insurer, the higher the price of the put option contingent on the non-tradeable risky asset $F$.

Example 4.4. Before we investigate the benefit stream (2.9) generated by unit-linked policies, let us consider the benefit stream generated by traditional insurance policies. Let us assume that the benefits $S$ and $D$ in (2.9) are constant. The premium is invested in the risk-free bank account and no fees are continuously deducted from the policyholder's account, i.e. $c=0$.

Let us study a single life insurance policy with constant sum insured $D$, paid if the policyholder dies. We choose $S=0, k=1$ in (2.9). The PDEs (4.8) reduce to one PDE:

$$
\begin{aligned}
& \varphi_{t}^{1}(t)+\left(D-\varphi^{1}(t)\right) \lambda+\frac{1}{2} \gamma \sqrt{\left|D-\varphi^{1}(t)\right|^{2} \lambda}-\varphi^{1}(t) r=0 \\
& \varphi^{1}(T)=0 .
\end{aligned}
$$


It is natural to assume that the sum at risk, i.e $D-\varphi^{1}(t)$, is positive at all times $t \in[0, T]$. Hence, we try to solve the PDE

$$
\begin{aligned}
& \varphi_{t}^{1}(t)+\left(D-\varphi^{1}(t)\right) \lambda\left(1+\frac{1}{2} \frac{\gamma}{\sqrt{\lambda}}\right)-\varphi^{1}(t) r=0 \\
& \varphi^{1}(T)=0 .
\end{aligned}
$$

The solution to 4.22 is given by

$$
\varphi^{1}(t)=D \int_{t}^{T} e^{-r(s-t)} e^{-\lambda\left(1+\frac{1}{2} \frac{\gamma}{\sqrt{\lambda}}\right)(s-t)} \lambda\left(1+\frac{1}{2} \frac{\gamma}{\sqrt{\lambda}}\right) d s .
$$

It is straightforward to show that $\varphi^{1}(t)<D$. Consequently, the function (4.23) also solves the PDE (4.21). Hence, (4.23) represents the continuous-time valuation operator for a single traditional life insurance policy.

Let us now study a single endowment insurance policy with constant terminal benefit $S$ paid if the policyholder survives till maturity. We choose $D=0, k=1$ in (2.9). The PDEs (4.8) reduce to

$$
\begin{aligned}
& \varphi_{t}^{1}(t)-\varphi^{1}(t) \lambda+\frac{1}{2} \gamma \sqrt{\left|\varphi^{1}(t)\right|^{2} \lambda}-\varphi^{1}(t) r=0 \\
& \varphi^{1}(T)=S .
\end{aligned}
$$

It is obvious that $\varphi^{1}$ is positive, and we deal with the $\mathrm{PDE}$

$$
\begin{aligned}
& \varphi_{t}^{1}(t)-\varphi^{1}(t) \lambda\left(1-\frac{1}{2} \frac{\gamma}{\sqrt{\lambda}}\right)-\varphi^{1}(t) r=0, \\
& \varphi^{1}(T)=S .
\end{aligned}
$$

The solution to 4.25$)$ is given by

$$
\varphi^{1}(t)=S e^{-r(T-t)} e^{-\lambda\left(1-\frac{1}{2} \frac{\gamma}{\sqrt{\lambda}}\right)(T-t)} .
$$

Hence, (4.26) is the continuous-time valuation operator for a single traditional endowment insurance policy. We notice that if we choose $\gamma$ arbitrary large, then $\varphi^{1}(t)>S e^{-r(T-t)}$, and $\varphi^{1}$ yields an arbitrage price of the insurance risk. Hence, when valuating endowment policies with the continuous-time valuation operator (4.8), we must restrict possible values of the risk aversion coefficient $\gamma$ in order to get reasonable, arbitrage-free prices.

Example 4.5. We continue Example 4.2. We derive the arbitrage-free representation (2.10) for the valuation operator $\varphi$ which solves the PDE (4.8). The arbitrage-free representation allows us to identify the risk premium for the measure (2.11) used for pricing the nonhedgeable insurance risk which is implied by our subjective valuation operator. 
Let $\tilde{\mathbb{Q}}$ denote the equivalent martingale measure defined by 2.11 with the risk premium

$$
\chi(t)=\frac{1}{2} \frac{\gamma}{\sqrt{J(t-) \lambda}} \operatorname{sgn}\left(\varphi^{J(t-)-1}(t, F(t))+D(F(t))-\varphi^{J(t-)}(t, F(t))\right) .
$$

If the process $\chi$ is smaller than -1 , then an equivalent martingale measure cannot be defined and our valuation operator 4.8 yields arbitrage prices of the insurance risk, see Example 4.4. Clearly, such cases should be eliminated. E.g. for bounded $\varphi$ and $D$, we can guarantee that $\chi(t)>-1$ by choosing $\gamma$ sufficiently small. Note that the process $\chi$ can be smaller than -1 only if $\varphi^{k-1}(t, f)+D(f)-\varphi^{k}(t, f)$ is negative, i.e. if the sum at risk is negative.

Let us assume that we can define the equivalent martingale measure $\tilde{\mathbb{Q}}$. By Girsanov's theorem, the counting process $N$ has the $\tilde{\mathbb{Q}}$-intensity

$$
\lambda^{\tilde{\mathbb{Q}}}(t)=\lambda\left(1+\frac{1}{2} \frac{\gamma}{\sqrt{J(t-) \lambda}} \operatorname{sgn}\left(\varphi^{J(t-)-1}(t, F(t))+D(F(t))-\varphi^{J(t-)}(t, F(t))\right)\right),
$$

and the dynamics of the policyholder's fund $F$ is given by

$$
\frac{d F(t)}{F(t)}=(r-c) d t+\sigma_{Y} d W_{Y}^{\tilde{\mathbb{Q}}}(t) .
$$

We can rewrite the system of PDEs 4.8) in the form

$$
\begin{aligned}
& \varphi_{t}^{k}(t, f)+\varphi_{f}^{k}(t, f) f(r-c)+\frac{1}{2} \varphi_{f f}^{k}(t, f) f^{2} \sigma_{Y}^{2}-k c f \\
& +\left(\varphi^{k-1}(t, f)+D(f)-\varphi^{k}(t, f)\right) \\
& \quad \cdot k \lambda\left(1+\frac{1}{2} \frac{\gamma}{\sqrt{k \lambda}} \operatorname{sgn}\left(\varphi^{k-1}(t, f)+D(f)-\varphi^{k}(t, f)\right)\right)-\varphi^{k}(t, f) r=0, \\
& \varphi^{k}(T, f)=k S(f) .
\end{aligned}
$$

Applying the Feynman-Kac formula, we can show that the solution of the PDEs 4.29, and also of the PDEs 4.8), has the representation

$$
\begin{aligned}
\varphi^{k}(t, f)= & \mathbb{E}_{t, f, k}^{\tilde{\mathbb{Q}}}\left[e^{-r(T-t)}(n-N(T)) S(F(T))+\int_{t}^{T} e^{-r(s-t)} D(F(s)) d N(s)\right. \\
& \left.-\int_{t}^{T}(n-N(s)) e^{-r(s-t)} c F(s) d s\right] .
\end{aligned}
$$

Under the valuation operator $\varphi$ determined by the PDEs (4.8), we price the unit-linked insurance claims contingent on $(F, N)$ with the expected value operator under the measure $\tilde{\mathbb{Q}}$ which distorts the distributions of the policyholders' lifetimes (the intensity of the counting process $N$ ). The policyholder's mortality intensity is modified under the pricing measure $\tilde{\mathbb{Q}}$ with the risk premium $\chi$ for which the sign depends on the sign of $\varphi^{k-1}(t, f)+D(f)-\varphi^{k}(t, f)$, that is the sum at risk. 
From (4.27) we can deduce that if the insurer is exposed to a positive sum at risk, then the process $\chi$ increases the mortality intensity, while if he is exposed to a negative sum at risk, then the process $\chi$ decreases the mortality intensity. Moreover, the risk premium $\chi$ for the insurance risk depends on the number of policies in force in the portfolio: the larger the number of policies in force, the smaller the risk premium, and the less significant the change in the policyholder's mortality intensity when we move from the real-world measure $\mathbb{P}$ to the pricing measure $\tilde{\mathbb{Q}}$. These properties agree with intuition, and the last property reflects diversification of the unsystematic mortality risk.

We would like to point out that under the equivalent martingale measure $\tilde{\mathbb{Q}}$, the insurance risk may not be independent of the financial risk and the policyholders' lifetime may not be independent of each other since $\chi$ in general depends on $F$. E.g. let us consider policies with minimum death guarantees. If the policyholder's fund $F$ is very low, then the insurer is exposed to a positive sum at risk, while if this fund is very high, then the insurer is not exposed to a sum at risk. Consequently, the insurer may prefer to increase the death probabilities in the first case, and keeps it unchanged in the second case. Moreover, since the policyholders own the same risky asset $F$, their mortality intensities and future lifetimes become correlated under the above mechanism of updating the model's parameters for pricing the future claims.

Let us investigate the minimal death guarantee (GMDB) for a single policy. Hence, we choose $S(t, f)=0$ and $k=1$ in the benefit stream (2.9). We also assume that no fees are continuously deducted from the fund $F$, i.e. $c=0$, and the guarantee is financed with the initial fee. We expect that the sum at risk is positive in all scenarios. We note that this assumption implies that the insurance risk is independent of the financial risk under $\tilde{\mathbb{Q}}$. From 4.27) and (4.30) we conclude that

$$
\begin{aligned}
\varphi^{1}(t, f) & =\int_{t}^{T} \mathbb{E}_{t, f}^{\hat{\mathbb{Q}}}\left[e^{-r(s-t)}\left(D^{*}-F(s)\right)^{+}\right] e^{-\lambda^{\tilde{\mathbb{Q}}}(s-t)} \lambda^{\tilde{\mathbb{Q}}} d s, \\
& =\int_{t}^{T} P\left(t, s, \sigma_{Y}, f, D^{*}\right) e^{-\lambda \tilde{\mathbb{Q}}(s-t)} \lambda^{\tilde{\mathbb{Q}}} d s
\end{aligned}
$$

where

$$
\lambda^{\tilde{\mathbb{Q}}}=\lambda\left(1+\frac{1}{2} \frac{\gamma}{\sqrt{\lambda}}\right) .
$$

Unfortunately, the sum at risk $D(f)-\varphi^{1}(t, f)$, where $\varphi^{1}$ is defined in 4.31, is not always positive for all parameters, in contrast to the traditional life insurance case considered in Example 4.4. However, in practice the guaranteed death benefit $D^{*}$ is very high compared 
to the fund value $F$. In this case one can show that

$$
\begin{aligned}
\varphi^{1}(t, f) & =\int_{t}^{T} P\left(t, s, \sigma_{Y}, f, D^{*}\right) e^{-\lambda^{\tilde{\mathbb{Q}}}(s-t)} \lambda^{\tilde{\mathbb{Q}}} d s \\
& \approx \int_{t}^{T}\left(D^{*} e^{-r(s-t)}-F(t)\right) e^{-\lambda^{\tilde{\mathbb{Q}}}(s-t)} \lambda^{\tilde{\mathbb{Q}}} d s \\
& \leq\left(D^{*}-F(t)\right) \mathbb{P}(\tau<T \mid \tau>t) \leq D^{*}-F(t),
\end{aligned}
$$

and the sum at risk, $D(f)-\varphi^{1}(t, f)$ with 4.31), is indeed positive. Consequently, we can conclude that the function (4.31) solves the PDE (4.8) with $k=1, S(f)=0, D(f)=$ $\left(D^{*}-f\right)^{+}, c=0$ and gives the price of the death guarantee from a single unit-linked insurance policy with high mortality benefit $D^{*}$.

From (4.9) we deduce the decomposition of the fair price:

$$
\begin{aligned}
\varphi^{1}(t, f)= & \mathbb{E}_{t, f}^{\hat{\mathbb{Q}}}\left[\int_{t}^{T} e^{-r(s-t)}\left(D^{*}-F(s)\right)^{+} e^{-\lambda(s-t)} \lambda d s\right] \\
& +\mathbb{E}_{t, f}^{\hat{\mathbb{Q}}}\left[\frac{1}{2} \gamma \int_{t}^{T} e^{-r(s-t)} \sqrt{\left|D(F(s))-\varphi^{1}(s, F(s))\right|^{2} \lambda} e^{-\lambda(s-t)} d s\right] .
\end{aligned}
$$

The best estimate of the liability, the first term in (4.33), is the expected value of the discounted future claim from the put option exercised at the random time of the policyholder's death, where the expected value is taken under the measure $\hat{\mathbb{Q}}$. This value can be related to the market cost of the investment portfolio which perfectly replicates the hedgeable part of the death guarantee, i.e. the payoff from the put option exercised at a pre-defined time. The timing of the benefit is not hedgeable since it is triggered by the non-tradeable, independent insurance risk. In the best estimate, the timing of the benefit payment is derived by taking the expected value of the future lifetime of the policyholder under the real-world measure $\mathbb{P}$. The best estimate in 4.33 is equal to

$$
B E=\int_{t}^{T} P\left(t, s, f, \sigma_{Y}, D^{*}\right) e^{-\lambda(s-t)} \lambda d s .
$$

In this example the insurer is exposed to changes in the tradeable asset price $Y$ which imply changes in the value of the policyholder's account $F$ and, consequently, changes in the price of the death guarantee embedded in the unit-linked insurance contract. The insurer is also exposed to the unpredictable event of the policyholder's death and the related timing of the death payment. By (4.6) the optimal hedging strategy consists of applying the deltahedging strategy

$$
\vartheta^{*}(t)=\varphi_{f}^{1}(t, F(t)) F(t) .
$$

By performing this strategy, the insurer perfectly hedges the changes in the price of the claim $\varphi$ resulting from changes in $Y$ and $F$, since $F$ is perfectly correlated with $Y$. However, 
the independent event of the policyholder's death and the timing of the payment cannot be hedged. From (4.10) we derive the dynamics of the net asset value

$$
\begin{aligned}
d N A V(s)= & N A V(s) r d t+\frac{1}{2} \gamma \sqrt{\left|D(F(s))-\varphi^{1}(s, F(s))\right|^{2} J(s-) \lambda} d s \\
& -\left(D(F(s))-\varphi^{1}(s, F(s))\right) d \tilde{N}(s) .
\end{aligned}
$$

The stochastic integral in 4.35 represents the sum at risk in case of a policyholder's death. At each time $t \in[0, T)$, the insurer must hold the additional capital in the amount of

$$
\Phi(t)=\frac{1}{2} \gamma \sqrt{\left|D(F(t))-\varphi^{1}(s, F(t))\right|^{2} J(t-) \lambda},
$$

to protect himself against adverse scenarios in the evolution of the non-hedgeable mortality risk in an infinitesimal period of time. The total actuarial risk margin, the second term in (4.33), is the expected total cost of providing sufficient capitals to cover the future sum at risk to which the insurer is exposed in the event of the policyholder's death. The capital in the amount of the sum at risk cannot be set aside by the insurer since this cost is too high to bear. However, the capital $\Phi$ is sufficient to cover the non-hedgeable insurance loss (in our model where the insurer uses a standard deviation risk margin for quantifying the non-hedgeable risks).

If the sum at risk is positive, then from 4.27) we can conclude that the mortality intensity under the pricing measure $\tilde{\mathbb{Q}}$ is an increasing function of the risk aversion coefficient $\gamma$, and from 4.30 we can conclude that the price $\varphi^{1}$ is an increasing function of the risk aversion coefficient $\gamma$. Consequently, the higher the risk aversion coefficient of the insurer, the higher the price of the minimum death guarantee determined by our continuous-time valuation operator (4.8). These interpretations agree with intuition.

We also investigate the minimum survival benefit (GMMB) for a single policy. We now choose $D(t, f)=0$ and $k=1$ in the benefit stream (2.9). We still assume that no fees are continuously deducted from the fund $F$, i.e. $c=0$, and the guarantee is financed with the initial fee. It is clear that the sum at risk is negative in all scenarios in this example. Hence, the sign of $\varphi^{0}(t, f)+D(t, f)-\varphi^{1}(t, f)$ can be determined, and the solution to the PDE (4.8) can be found. From the general solution 4.30 we can deduce that the price of the survival guarantee is given by

$$
\begin{aligned}
\varphi^{1}(t, f) & =\mathbb{E}_{t, f}^{\hat{\mathbb{Q}}}\left[e^{-r(T-t)}\left(S^{*}-F(T)\right)^{+}\right] e^{-\lambda^{\tilde{\mathbb{Q}}}(T-t)} \\
& =P\left(t, T, \sigma_{Y}, f, S^{*}\right) e^{-\lambda^{\tilde{\mathbb{Q}}}(T-t)}
\end{aligned}
$$

where

$$
\lambda^{\tilde{\mathbb{Q}}}(t)=\lambda\left(1-\frac{1}{2} \frac{\gamma}{\sqrt{\lambda}}\right) .
$$


By Example 4.4, we have to choose a sufficiently small risk aversion coefficient $\gamma$ in order to have an arbitrage-free price (4.38). By (4.9) we can decompose the fair price of the liability into the best estimate of the liability and the total actuarial risk margin:

$$
\begin{aligned}
\varphi^{1}(t, f)= & \mathbb{E}_{t, f}^{\hat{\mathbb{Q}}}\left[e^{-r(T-t)}\left(S^{*}-F(T)\right)^{+}\right] e^{-\lambda(T-t)} \\
& +\mathbb{E}_{t, f}^{\hat{\mathbb{Q}}}\left[\frac{1}{2} \gamma \int_{t}^{T} e^{-r(s-t)} \sqrt{\left|\varphi^{1}(s, F(s))\right|^{2} \lambda} e^{-\lambda(s-t)} d s\right] .
\end{aligned}
$$

Finally, from (4.10) we derive the dynamics of the net asset value

$$
d N A V(s)=N A V(s) r d t+\frac{1}{2} \gamma \sqrt{\left|\varphi^{1}(s, F(s))\right|^{2} J(s-) \lambda} d s+\varphi^{1}(s, F(s)) d \tilde{N}(s) .
$$

The hedging strategy takes the form (4.34).

The interpretations of the results are similar as in the previous example with death guarantee. However, we should note that the stochastic integral in 4.39 now models the unpredictable mortality gains to which the insurer is exposed during the duration of the policy. Since we consider an endowment policy, mortality gain arises if the policyholder dies. We note that we deal with the dynamics

$$
\begin{aligned}
d N A V(s)= & N A V(s) r d t+\frac{1}{2} \gamma \sqrt{\left|\varphi^{1}(s, F(s))\right|^{2} J(s-) \lambda} d s \\
& -\varphi^{1}(s, F(s)) J(s-) \lambda d s+\varphi^{1}(s, F(s)) N(s),
\end{aligned}
$$

and the insurer gains if $d N(s)=1$. At each time $t \in[0, T)$, the insurer holds an additional capital $\Phi(t)=\frac{1}{2} \gamma \sqrt{\left|\varphi^{1}(t, F(t))\right|^{2} J(t-) \lambda}$ to protect himself against adverse scenarios in the non-hedgeable longevity risk, i.e. in this case the risk that policyholders live longer than expected.

\section{$5 \quad$ Numerical examples}

This section reports some numerical examples illustrating the developments of the previous sections.

\subsection{Comparison of the explicit and approximate solution for a GMDB contract}

In the previous section, we have seen that the function (4.31) solves the PDE (4.8) for a single unit-linked insurance policy with a minimum death guarantee excluding fees when the mortality guaranteed benefit $D^{*}$ is set sufficiently high. We illustrate that the function (4.31) indeed closely approximates the true fair value of the policy if the mortality benefit 
$D^{*}$ is set high compared to the initial fund value $F(0)$, whereas the function (4.31) provides a (relatively) poor approximation of the solution to the PDE (4.8) when the mortality benefit $D^{*}$ is set low compared to the initial fund value $F(0)$. Hence, one needs to be careful when applying the price (4.31) as the solution to the PDE (4.8) and the fair value of the GMDB contract.

Figure 1 illustrates the fair value at time $t=0$ for the single GMDB contract in terms of the risk aversion parameter $\gamma$. The fair value is derived by solving numerically the PDE (4.8) with a finite difference scheme. In addition, the approximation price (??) is provided. We can observe that the difference between both approaches is quite significant for a low mortality benefit $\left(D^{*}=12>F(0)=11\right)$, whereas the prices coincide for a high mortality benefit $\left(D^{*}=20>>F(0)=11\right)$. As expected, the fair value is an increasing function of the risk aversion parameter.

\subsection{Sensitivity analysis for a single unit-linked contract with death and survival benefits}

In this numerical example, we determine the fair value of a single unit-linked policy with death and survival benefits and observe how the fair value is sensitive to the numerical values chosen for the parameters. We set $k=1, D(f)=\left(D^{*}-f\right)^{+}, S(f)=\left(S^{*}-f\right)^{+}$in 4.8). We solve the PDE (4.8) numerically by applying a finite difference scheme.

Figure 2 depicts the fair value of the contract at time $t=0$ in terms of the risk aversion parameter $\gamma$, the mortality intensity $\lambda$, the volatility parameter $\sigma$ and the fee $c$. We observe the following phenomena:

- The fair value is an increasing function of $\gamma$. Indeed, the risk aversion is directly related to the actuarial risk margin for the non-hedgeable insurance risk. Increasing the risk aversion parameter $\gamma$ is equivalent to increasing the risk margin for the uncertainty around the policyholder's death. The higher the risk aversion parameter, the higher the price of the non-hedgeable insurance risk determined by the instantaneous actuarial risk margin, and the higher the value of the unit-linked contract.

- The fair value is an increasing function of $\lambda$. Intuitively, when the mortality increases, the value of the GMDB increases while the value of the GMMB decreases. Since this unit-linked contract offers both death and survival benefits, the figure shows that the GMDB dominates the GMMB for our choice of parameters. If we decrease the death benefit, the trend will be reversed.

- The fair value is an increasing function of $\sigma$. This follows from the observation that more volatility in the financial market boosts the prices of the put options for the death 
and survival guaranteed benefits yielding a higher value of the unit-linked contract.

- The fair value is a decreasing function of $c$. The higher the fee deducted from the policyholder's account, the higher the profit earned by the insurer and the lower the gains earned by the underlying risky asset credited to the policyholder's account which determine the benefits. As Figure 2 shows, the fair value of the unit-linked contract can be negative if the fee $c$ deducted by the insurer from the policyholder's account is too high compared to the value of the risk faced by the insurer. The fair fee is the fee for which the fair value of the benefit stream at the inception of the contract is zero.

\subsection{Fair fee $c$ for a GMMB contract and sensitivity analysis}

In this subsection we determine the fair fee $c$ for a single GMMB contract and perform a sensitivity analysis for changes in the mortality and volatility parameters. Starting from the general representation 4.30, we can derive the expression for the fair value of a single GMMB contract:

$$
\varphi^{1}(t, f)=\mathbb{E}_{t, f, 1}^{\tilde{\mathbb{Q}}}\left[e^{-r(T-t)} \mathbf{1}\{\tau>T\}\left(S^{*}-F(T)\right)^{+}-\int_{t}^{T} \mathbf{1}\{\tau>s\} e^{-r(s-t)} c F(s) d s\right] .
$$

This expression reduces to

$$
\varphi^{1}(t, f)=e^{-\lambda^{\tilde{\mathbb{Q}}}(T-t)} e^{-c(T-t)} P\left(t, T, f, \sigma_{Y}, S^{*} e^{c(T-t)}\right)-c f \int_{t}^{T} e^{-c(s-t)} e^{-\lambda^{\tilde{\mathbb{Q}}}(s-t)} d s .
$$

where

$$
\lambda^{\tilde{\mathbb{Q}}}=\lambda\left(1-\frac{1}{2} \frac{\gamma}{\sqrt{\lambda}}\right)
$$

We remark that the price $(5.2)$ has the same structure as the price $(3.8)$, the difference being that in $(5.2)$ the insurance risk is priced with a risk margin, i.e. $\lambda^{\tilde{\mathbb{Q}}}$ instead of $\lambda$, while in (3.8) the insurance risk follows the expected mortality $\lambda$.

Since the continuously collected fee $c$ is used to hedge the GMMB, we determine the fair

fee $c$ by setting the fair value at time $t=0$ in $(5.2)$ equal to zero (expected future benefits equal expected future fees). Hence, this boils down to solving the following equation:

$$
e^{-\left(\lambda^{\tilde{\mathbb{Q}}}+c\right) T} P\left(0, T, F(0), \sigma_{Y}, S^{*} e^{c T}\right)=\frac{c F(0)}{\lambda \tilde{\mathbb{Q}}+c}\left(1-e^{-\left(\lambda^{\tilde{\mathbb{Q}}}+c\right) T}\right) .
$$

Figure 3 represents the fair fee $c$ in terms of the mortality parameter $\lambda$ and the volatility parameter $\sigma_{Y}$ : 
- The fair fee $c$ is a decreasing function of the mortality intensity $\lambda$. When the mortality intensity increases, this diminishes the survival probability until maturity and the insurer therefore asks a lower fee for the maturity survival guarantee.

- The fair fee $c$ is an increasing function of the volatility parameter $\sigma_{Y}$. As in Section 5.2 , a more volatile financial market is synonymous with a higher price of the put option $\left(S^{*}-F(T)\right)^{+}$that the policyholder will receive in case of survival. Hence, the higher the volatility in the financial market, the higher fair fee for financing the maturity survival guarantee.

\section{References}

Barigou, K. \& Dhaene, J. (2018), 'Fair valuation of insurance liabilities via mean-variance hedging in a multi-period setting', Scandinavian Actuarial Journal .

URL: https://doi.org/10.1080/03461238.2018.1528477

Delong, Ł. (2013), Backward Stochastic Differential Equations with Jumps and Their Actuarial and Financial Applications, Springer.

Delong, Ł., Dhaene, J. \& Barigou, K. (2018), 'Fair valuation of insurance liability cash-flow streams in continuous time: Theory'. Submitted.

Dhaene, J., Stassen, B., Barigou, K., Linders, D. \& Chen, Z. (2017), 'Fair valuation of insurance liabilities: merging actuarial judgement and market-consistency', Insurance: Mathematics and Economics 76, 14-27.

Happ, S., Merz, M. \& Wüthrich, M. V. (2015), 'Best-estimate claims reserves in incomplete markets', European Actuarial Journal 5(1), 55-77.

Pelsser, A. (2010), 'Time-consistent and market-consistent actuarial valuations'. Available at SSRN: https://ssrn.com/abstract $=1551323$.

Pelsser, A. \& Ghalehjooghi, A. S. (2016), 'Time-consistent actuarial valuations', Insurance: Mathematics and Economics 66, 97-112. 

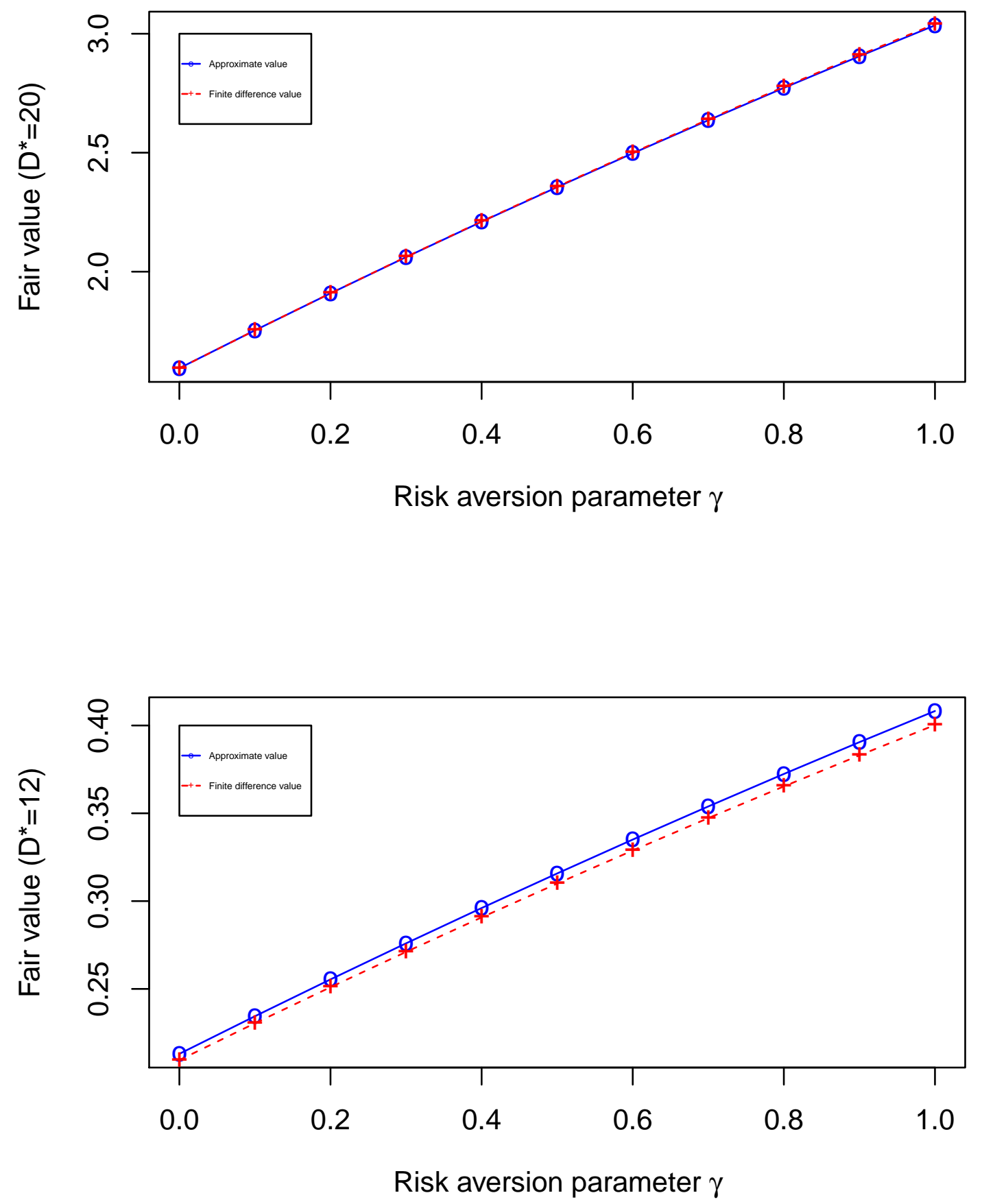

Figure 1: Comparison of the finite difference solution to the PDE (4.8) and the approximate solution (??) for a single GMDB with high death benefit $D^{*}=20$ and low death benefit $D^{*}=12$. We choose $r=0.02, \sigma=0.2, \lambda=0.2, F(0)=11, T=1$. 


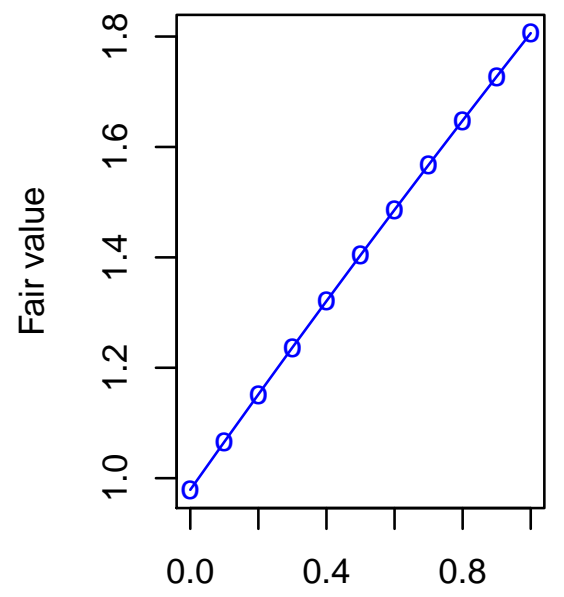

Risk aversion parameter $\gamma$

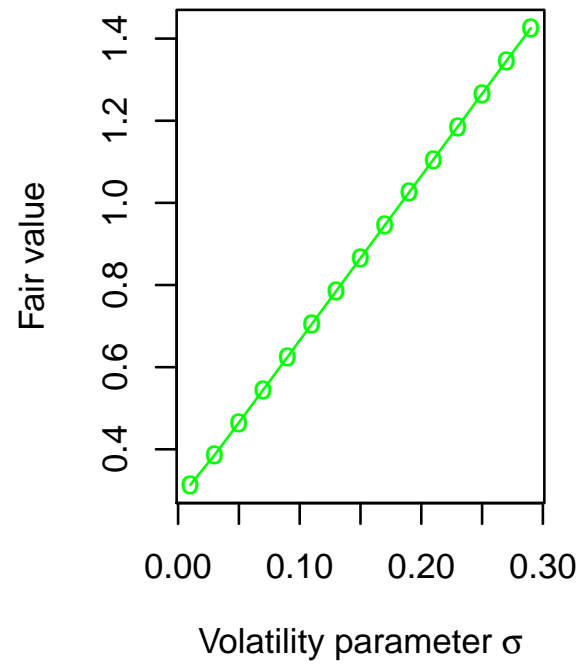

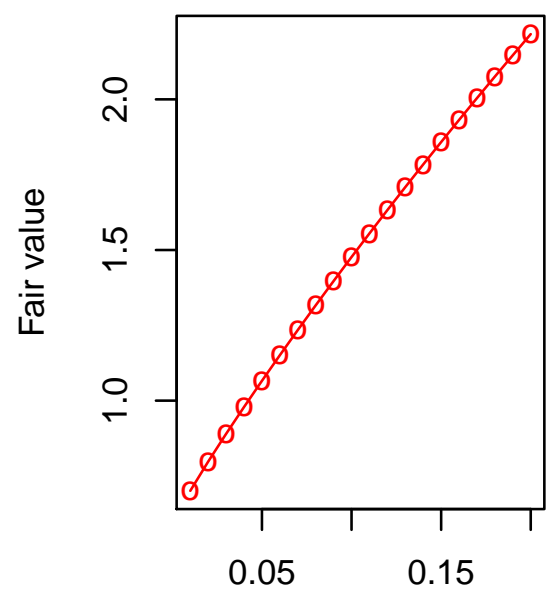

Mortality parameter $\lambda$

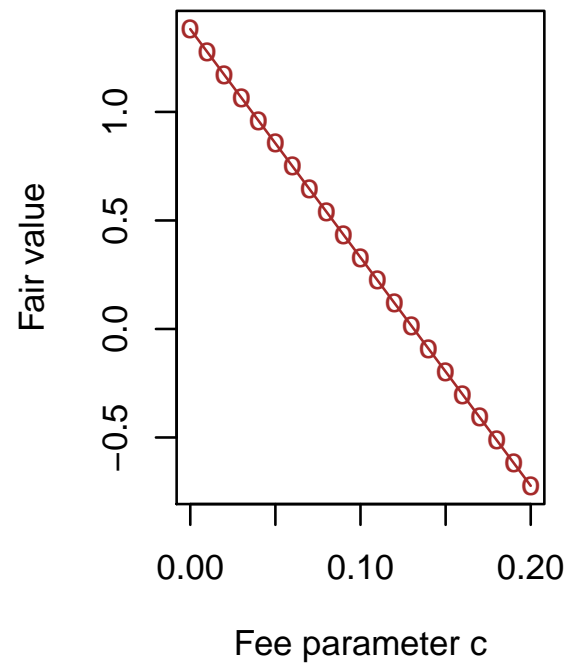

Figure 2: Sensitivity of the fair value of a single unit-linked contract with respect to risk aversion, mortality, volatility and fee parameters. We choose $r=0.02, c=0.03, \sigma=0.2, \lambda=$ $0.05, F(0)=S^{*}=11, D^{*}=20, \gamma=0.1, T=1$. 

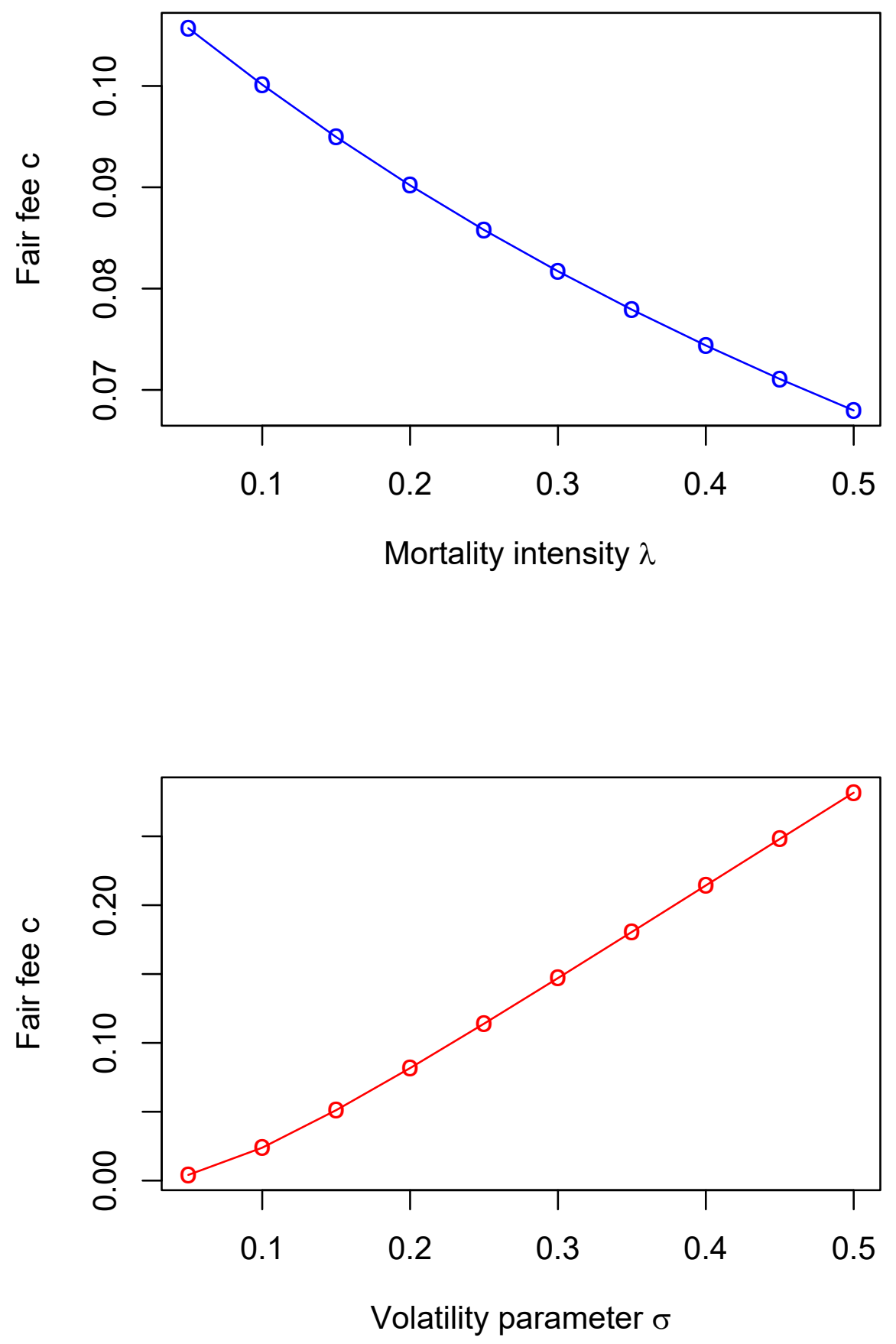

Figure 3: Sensitivity of the fair fee for a single GMMB contract with respect to mortality and volatility parameters. We choose $r=0.02, \sigma=0.2, \lambda=0.3, F(0)=S^{*}=11, \gamma=0.1, T=1$. 